\title{
Detecting changes between real-world objects using spatiochromatic filters
}

\author{
GREGORY J. ZELINSKY \\ State University of New York, Stony Brook, New York
}

\begin{abstract}
A behavioral and computational treatment of change detection is reported. The behavioral task was to judge whether a single object substitution change occurred between two "flickering" 9 -object scenes. Detection performance was found to vary with the similarity of the changing objects; object changes violating orientation and category yielded the fastest and most accurate detection responses. To account for these data, the BOLAR model was developed, which uses color, orientation, and scale selective filters to compute the visual dissimilarity between the pre- and postchange objects from the behavioral study. Relating the magnitude of the BOLAR difference signals to change detection performance revealed that object pairs estimated as visually least similar were the same object pairs most easily detected by observers. The BOLAR model advances change detection theory by (1) demonstrating that the visual similarity between the change patterns can account for much of the variability in change detection behavior, and (2) providing a computational technique for quantifying these visual similarity relationships for real-world objects.
\end{abstract}

Our ability to detect change between real-world scenes is rich in information about how we represent the visual world. Of particular recent interest has been the information also available from change detection failures, cases in which observers are seemingly "blind" to the occurrence of a change (Rensink, 2002; Simons \& Levin, 1997). Whereas correct change detection judgments imply a successful representation and comparison of the objects undergoing change, systematic failures to detect change may indicate weaknesses or holes in a representational structure.

In the present study, I use behavioral and imageprocessing techniques to explore the representational constraints revealed by a change detection task. I will first discuss three forms of representational limitation likely to affect change detection behavior, loosely characterized as memory-, attention-, and similarity-based constraints. I will argue that renewed attention should be paid especially to the visual similarity relationships between the objects undergoing change. Second, I will explore the theoretical implications that these various forms of constraint have for capacity-limited models of change detection, arguing that higher level processes or

I thank George McConkie and Gary Wolverton for the stimulus presentation environment used in the behavioral experiments, and Narayan Srinivasa and Felix Chi for their help implementing the computational model. I also thank Dave Irwin, Lester Loschky, and George McConkie for many insightful comments on and discussions of change detection that profoundly influenced my thinking. This project was supported in part by NSF Grant ITR 0082602, and an abbreviated version of this work was presented at the 1999 meeting of the Association for Research in Vision and Ophthalmology. Address correspondence to G. J. Zelinsky (gzelinsky@ notes.cc.sunysb.edu) at the Department of Psychology, Psych B Bldg., Room 240, SUNY, Stony Brook, NY 11794-2500. parameters should be assumed only after similarity-based constraints have been found inadequate for explaining the observed data. Third, I will report a behavioral experiment demonstrating a similarity-based constraint, one involving an effect of orientation and categorical similarity on change detection. Last, I will outline an imagebased representational substrate capable of capturing these effects of visual similarity on real-world change detection performance. This final contribution of the study is particularly important in that a simple technique is described that can be used to quantify the visual similarity relationships between pre- and postchange patterns regardless of their featural complexity. Moreover, this technique is framed in terms of a computational model. Despite the prominent use of realistic stimuli in the change detection literature, no computationally explicit model has been demonstrated to work for real-world changes. The present work is intended to help bridge this gap between change detection theory and the stimuli commonly used to investigate this behavior.

\section{Sources of Representational Constraint in a Change Detection Task}

The representational weaknesses revealed by change detection failure are described most often in terms of memory and attentional constraints. Memory-based detection failures will result if the objects undergoing change are not represented in an enduring form (Grimes, 1996; O'Regan, 1992), or if a coherent representation of the prechange object does not exist at the time of the postchange comparison (Rensink, 2000a). Memory might also constrain performance if the number of objects in the prechange scene exceeds a capacity limit imposed by working memory (but see Rensink, 2000b), or if the build-up 
of retroactive interference during scene viewing restricts confident retrieval to only the most recently processed objects (Zelinsky \& Loschky, 1998, 2003). Such limitations would result in good memory for only a subset of the scene's objects. The substitution of a can of soda with a glass of juice in a kitchen scene might therefore go unnoticed if the soda was not among this subset of accessible prechange objects in working memory. Related to memory constraints, attentional limitations are imposed on a change detection task by the serial encoding of scene objects into memory. Observers will inevitably find some aspects of a prechange scene more interesting or important than others, meaning that these scene objects will be preferentially selected for encoding. Attention-based constraints result when the time required for one to encode all of the scene's objects exceeds the presentation duration of the prechange display. In this event, "less important" scene objects will not benefit from detailed processing, thereby causing holes in the representational structure that might result in change detection failure (Henderson \& Hollingworth, 1999; Levin \& Simons, 1997; Rensink, O’Regan, \& Clark, 1997; Simons \& Levin, 1998). ${ }^{1}$

A third source of representational constraint stems not from an encoding limitation, but rather from the representational similarity between the changing objects. Both the can of soda and the glass of juice may be fully represented and available for comparison, but a change between these objects might still be difficult to detect if they had a great deal of representational overlap. The existence of such similarity-based constraints is supported by multidimensional scaling studies showing a relationship between the proximity of items in psychological space and the ability to correctly retrieve these items from memory (Shepard, 1980, 1987), as well as several investigations more targeted to a change detection task (ScottBrown, Baker, \& Orbach, 2000; Scott-Brown \& Orbach, 1999; Williams \& Simons, 2000; Zelinsky, 1998, 1999, 2001a).

The question of how best to quantify the visual similarity between real-world stimuli poses a difficult representational challenge to an understanding of change detection behavior. In most attempts to address this problem, researchers have hand picked a small number of featural dimensions on which to characterize the change stimuli. For example, Rensink et al. (1997) informally equated changes for color and luminance yet still observed a detection advantage for objects rated as important to the overall context of the scene. More recently, Simons, Franconeri, and Reimer (2000) coded the changes between fully realistic scenes in terms of size, contrast, and color differences. Size was quantified as the ratio of the change region, in pixels, to the total size of the image. Contrast was estimated by comparing the changed region to an equal-sized region immediately surrounding the changed object. Color change was quantified by first converting the original and modified scenes from RGB to $\mathrm{L}^{*} \mathrm{u}^{*} \mathrm{v} *$ coordinates, and then computing the Euclid- ean distance for each changed pixel in the image pair. Using these measures, the authors found no reliable correlations between change detection performance and change magnitude, and they concluded from these findings that detection is not meaningfully influenced by the magnitude of the visual change signal.

Care should be taken, however, not to dismiss a relationship between visual similarity and change detection performance on the basis of these negative results. The possibility remains that the stimulus dimensions chosen by these authors may not have been the most relevant to a change detection task, or that the methods used to quantify these visual properties may simply not have been sensitive enough to capture an effect of visual similarity on change detection performance. For example, the fairly gross visual similarity measures used in Simons et al.'s (2000) study may have missed many important visual properties contributing to the detection of change. Most notably absent from their study was any quantification of spatial structure in the changing patterns. By defining their size estimation solely in terms of the number of pixels constituting the change, they ignored the role that edge and contour information may play in the detection process.

The primary goal of the present study is to more thoroughly investigate the role of visual similarity in a change detection task. Rather than characterize the change stimuli in terms of a small number of hand-picked properties, I use an image-based technique to represent dozens of potentially relevant visual features. This approach has three clear advantages over alternative methods of quantifying visual similarity. First, such a high-dimensional feature representation has a greater chance of capturing the properties of the pre- and postchange patterns that are sensitive to a visual similarity manipulation-assuming that visual similarity plays any role at all in change detection. Second, this more extensive quantification of visual similarity provides a clearer estimate of similarity-based constraints on detection performance, thereby creating a baseline against which the added contribution of memory and attentional constraints can be meaningfully compared. Third, a filter-based approach can be applied to arbitrary stimuli ranging in complexity from oriented bars to fully realistic scenes. Such coding of change stimuli in a common featural space makes it possible to meaningfully compare the findings from a wider range of change detection studies.

\section{Implications of Change Detection Constraint on Capacity Limited Processing}

Explanations of change detection failure appealing to memory or attentional constraints implicitly assume the existence of one or more processing "bottlenecks" that ultimately limit the number of objects that can be encoded from a scene and used in a change detection task. The assumption of a processing bottleneck has profound consequences for change detection theory. By assuming that a bottleneck is required in order for one to explain 
change detection performance, one is also making a strong and direct claim against the existence of models that make no such assumptions. Relaxing a memory constraint on change detection would mean no longer assuming an a priori limit on the number of objects that could be encoded from a prechange scene and compared with a postchange scene. Relaxing an attentional constraint would mean that the encoding of objects into memory would not be limited by a serial process. Although such an unlimited-capacity parallel model of change detection seems implausible and indeed is probably inadequate for explaining the entirety of change detection behavior, in building a theory it is important to start with a model having the fewest possible number of assumed processes or parameters, and then to add parameters as required in order to explain new empirical data. If a highly parameterized theory is proposed before all simpler theories have been ruled out, one will never know whether the assumed processes are necessary to the explanation of the behavior in question. It is within this context that I believe the evidence against unlimitedcapacity theories of change detection, and for memory and attention bottlenecks, should be reevaluated.

There exists no critical study pitting bottleneck theories against unlimited-capacity parallel models of change detection. Instead, unlimited-capacity parallel models have been largely dismissed on the basis of evidence from two related topics of investigation. First, people do not form a composite visual pattern from two component patterns viewed in successive eye fixations (Bridgeman \& Mayer, 1983; Irwin, Brown, \& Sun, 1988; Rayner \& Pollatsek, 1983; though see Brockmole, Wang, \& Irwin, 2002). Furthermore, reading is apparently unaffected when the letters of words are alternated between upper and lowercase characters during eye movements (McConkie \& Zola, 1979). If detailed visual representations were available for use during these tasks, one might expect greater access to composite patterns and greater interference from case changes during reading. A second line of evidence against an unlimited-capacity parallel process comes directly from the change detection literature. Changes made to objects in scenes frequently go undetected, regardless of whether these changes have occurred during eye movements (Currie, McConkie, CarlsonRadvansky, \& Irwin, 2000; Grimes, 1996; Henderson \& Hollingworth, 1999; Hollingworth \& Henderson, 2000; McConkie \& Currie, 1996) or at some other time during a trial when the motion transient accompanying the change would likely be masked (Blackmore, Brelstaff, Nelson, \& Troscianko, 1995; Levin \& Simons, 1997; O’Regan, Rensink, \& Clark, 1999; Pashler, 1988; Rensink et al., 1997; Simons \& Chabris, 1999). In one of the few discussions explicitly addressing the implications of these detection failures for an unlimited-capacity model of change detection, Rensink (2000a) writes:

This "change blindness" suggests that little detailed information is being accumulated-otherwise, change detection would be easy, either by comparing immediate visual input with the contents of the buffer, or by detecting the anomalous structures formed by superimposing the original and changed images. (Rensink, 2000a, p. 18)

According to Rensink (2000a), the very existence of change detection failures therefore argues against an unlimited-capacity parallel comparison of the change stimuli. Presumably, if scenes were being represented and compared in parallel, any changes between these scenes would necessarily be signaled and detection failures should not occur.

Perhaps the strongest argument for a processing bottleneck comes from another study by Rensink in which both presentation duration and number of items were manipulated in a "flicker" change detection task (Rensink, 2000b; see also Zelinsky, 2001a). The displays consisted of 2,6 , or 10 oriented black or white rectangles, and presentation varied from 80 to $800 \mathrm{msec}$. A 120 -msec blank interval separated the presentations of each repeating stimulus display. Rensink (2000b) found a large effect of set size in this task that interacted with presentation duration and change type. When the task involved the detection of a horizontal-vertical orientation change, the set size effect was relatively constant over the 80- to 600 -msec range, but it increased in the $800-\mathrm{msec}$ presentation condition. Presentation duration had no effect on set size when the task involved the detection of polarity change (a black rectangle changing with a white rectangle). Rensink (2000b) concluded from these findings that detection performance is limited by an attentional process when displays are presented for durations shorter than $600 \mathrm{msec}$. He also concluded that detection performance with longer presentation durations, at least in the case of the orientation change task, is limited by memory constraints. By drawing a functional equivalence between the number of display items receiving attentional processing (as inferred from the reaction time $\times$ set size function) and the number of items that can be retained in memory, Rensink estimated that only 5.5 oriented items can be held in memory from one display presentation to the next. ${ }^{2}$ A larger memory capacity estimate of 9 items was obtained for the polarity detection task. Rensink attributed this discrepancy between the two capacity estimates to grouping factors inflating memory capacity when the detection task involved polarity change.

So persuasive were the arguments above that explanations of change detection suggesting a capacity-unlimited and parallel comparison of the pre- and postchange scenes have not been given serious consideration (though see Feldman, 1985, and McConkie \& Rayner, 1976). This relative neglect, however, is unfortunate, because each of these arguments offers only indirect evidence in support of memory or attentional bottlenecks. For example, the fact that people do not exploit a high-capacity visual buffer to integrate information across an eye movement in no way implies the absence of such a buffer. Failure to find evidence for the formation of composite patterns may instead reflect an inability to perceptually integrate two such buffers - an operation not required by change de- 
tection. Similarly, unless one adopts the awkward and computationally implausible assumption that a currently viewed scene is continuously compared in an obligatory fashion with a previously viewed scene regardless of the ongoing task, failing to find an effect of case changes on reading tells us precious little about the visual representations used by change detection. Even Rensink's (2000b) evidence for attention- and memory-based constraints on change detection is open to interpretation. Given arguments developed in the visual search literature (Eckstein, 1998; J. Palmer, Ames, \& Lindsey, 1993; Townsend, 1976, 1990), set size effects in a change detection task do not imply the existence of a serial attentional bottleneck. In fact, Zelinsky (2001a) interpreted a very similar pattern of data in terms of an unlimited-capacity parallel model. According to this interpretation, the addition of each item to a change detection display adds visual noise to the task of detecting a pattern change, noise that might shift the observer's decision criteria and potentially delay a response (though see Wright, Green, \& Baker, 2000).

As for Rensink's (2000a) claim that the very existence of change detection failure implies a sparse visual representation of the change stimuli, this argument suffers from a logical flaw. Although Rensink is quite correct to point out that a parallel process would necessarily generate a change signal, it does not follow that this signal would be sufficient to mediate detection. There are at least two reasons why a change signal might not result in a positive detection judgment. First, the visual representations used by change detection are likely to be constrained by the limitations of visual acuity (Henderson \& Hollingworth, 1999; Zelinsky, 2001a) and degraded by internal noise (Lu \& Dosher, 1999). Whereas the existence of a change signal might imply detection for an ideal noise-free observer, change detection in the real world occurs under far less ideal conditions. Second, all change signals are probably not created equal, with some changes producing larger signals than others. For example, large stimulus displacements made during a saccadic eye movement are easier to detect than small displacements (Bridgeman, Hendry, \& Stark, 1975; McConkie \& Currie, 1996), changes violating object type or semantic boundaries are detected better than token changes between semantically consistent objects (Friedman, 1979; Hollingworth \& Henderson, 2000; R. E. Parker, 1978), and changes to visually similar complex patterns are more difficult to detect than changes involving less similar patterns (Scott-Brown et al., 2000; Scott-Brown \& Orbach, 1999; Williams \& Simons, 2000; Zelinsky, 1998, 1999, 2001a). In addition to memory and attentional constraints, change detection therefore seems also to depend not only on memory and attentional constraints, but also on the magnitude of the change signal, with this magnitude being determined by the conceptual and visual similarity of the objects undergoing change. This relationship between signal strength and detection performance should come as no surprise. Change detection, like any other detection task, presupposes the successful differentiation of a signal from noise. To the extent that this is the case, even a parallel model free of memory and attentional limitations might produce change detection failures when the signal is weak or the noise too pronounced.

A secondary goal of the present study is to reexamine the potential of an unlimited-capacity parallel model to produce change detection behavior, and to argue that such a model may have been dismissed a bit too hastily. To make this argument, I adopt an integrated behavioral and computational approach. In Experiment 1, I report behavioral data from a "flicker" change detection task that cannot be easily explained by attentional or memory constraints limiting the processing of objects appearing in a given scene. In Experiment 2, I borrow filter-based image processing techniques from the vision science community and apply this low-level method of visual analysis to the task of change detection. Using these techniques, I show how a simple unlimited-capacity parallel model, one that compares low-level visual buffers of filter responses, can describe much of the change detection behavior observed in Experiment 1. Note, however, that neither of these behavioral or computational efforts in any way proves that change detection actually uses such an unlimited-capacity parallel process. This stronger claim would require manipulations and analyses that were not part of the present investigation. Rather, my intention here is to simply (1) provide an existence proof demonstrating the feasibility of an unlimitedcapacity parallel model to produce change detection behavior and (2) establish a visual similarity-derived baseline against which the need for additional memory and attentional constraints can be properly evaluated. By outlining a change detection theory constrained only by the similarity relationships between the objects undergoing change, I hope that the present study will highlight the need for critically justifying the use of additional parameters to explain change detection behavior.

\section{EXPERIMENT 1 \\ Effects of Orientation and Category Violation on Change Detection}

Change detection studies using fully realistic scenes or real-world events do not always control for all of the stimulus factors that might affect performance. For example, when one object was substituted with another in the Levin and Simons (1997) study, how closely did the two objects match in terms of color, size, and orientation? Failure to provide this information is an understandable omission when one is working with real-world objects, but it is nevertheless unfortunate. If a parallel process is used to compare one scene to another, the change-defining difference signal generated by this process will likely vary with the degree of mismatch along these featural dimensions. Another often overlooked factor in real-world change detection studies is the number of objects appearing in the change displays. Depending 
on one's definition of an object, realistic scenes might easily require the observer to monitor hundreds of patterns in their search for the change target (Zelinsky, 2001a). Given that the number of patterns requiring inspection might have profound consequences for hypothesized sources of change detection constraint, stimuli should also be characterized along this dimension. To address these concerns, I constructed a simpler class of pseudo-realistic scene stimuli consisting of nine visually distinct real-world objects appearing against a complex background. Each of the objects in these simple scenes was specified in terms of orientation and category. The present experiment asked how the orientation and categorical similarity between two real-world objects undergoing change might affect detection performance. To the extent that performance is driven by a similarity-based change signal, change detection should improve with the orientation and category mismatch between the changing objects.

\section{Method}

Participants. Ten SUNY Stony Brook graduate students were compensated $\$ 6 / \mathrm{h}$ for participating in this experiment. All of the observers were naive to the specif ic questions under investigation and had normal or corrected-to-normal visual acuity.

Stimuli. The stimuli were pseudo-realistic color scenes depicting 9 objects (tools or toys) arranged on a background surface (either a workbench or a crib). Twenty objects were used (10 tools and 10 toys), although only 9 appeared in any given scene. The toy and tool objects were of roughly uniform size and could each fit within an imaginary $2.4^{\circ}$ bounding box. The locations of the 9 objects appearing in each scene were randomly selected from 18 possible display positions forming an incomplete grid surrounding initial fixation. The composite scenes (objects and background) subtended $18^{\circ} \times 11.6^{\circ}$ of visual angle at a viewing distance of $82 \mathrm{~cm}$ and were of near photographic quality.

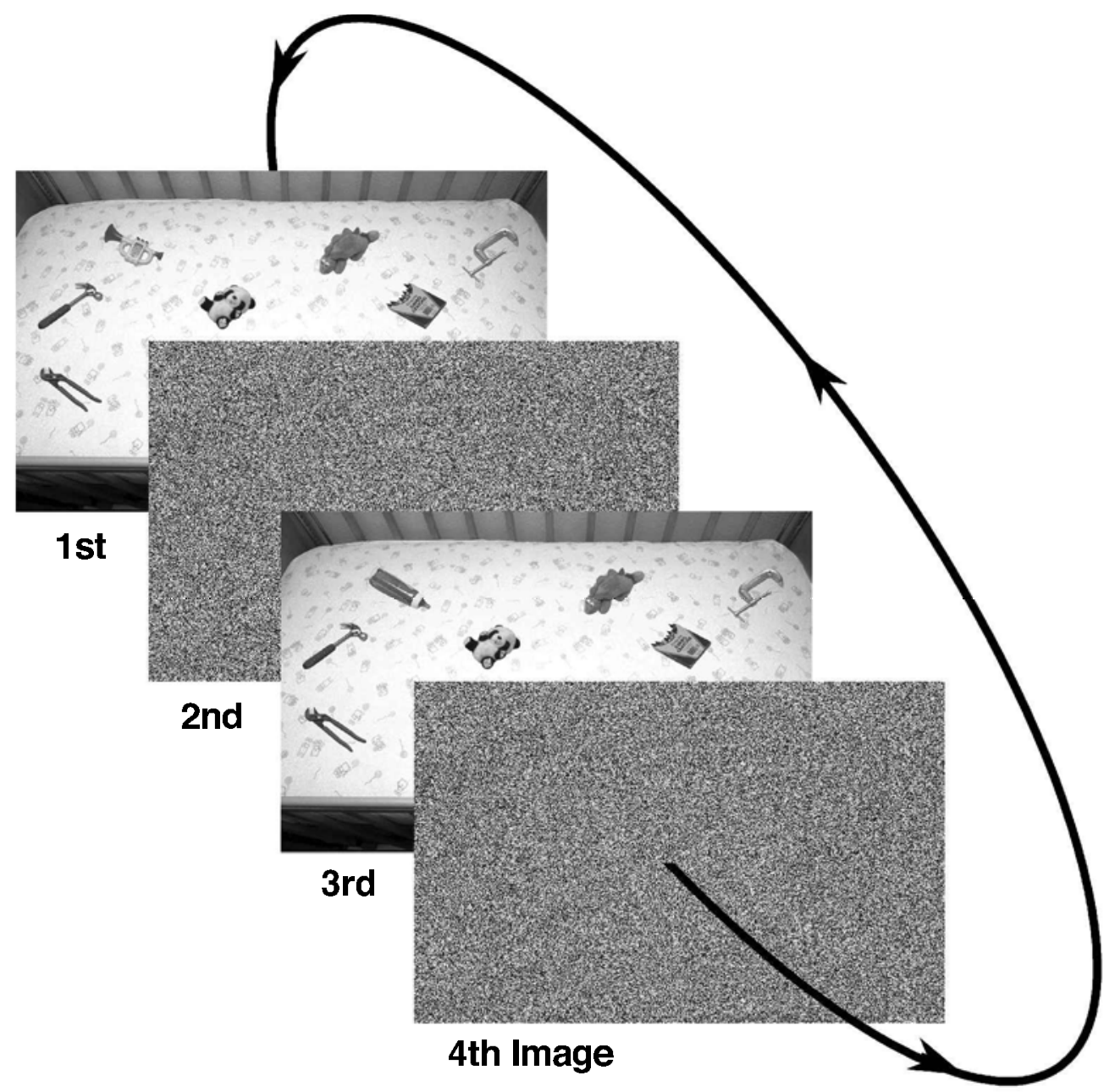

Figure 1. An example of the repeating sequence of stimuli constituting each experimental trial. One object pair, in this case the trumpet and baby bottle, repeatedly changed between images 1 and 3 on half of the trials. The scenes were identical on the remaining trials. Sandwiched between each scene was a noise mask. All four images were presented for $300 \mathrm{msec}$, with this sequence repeating until a buttonpress. Note that the actual images used in this study were in color. 
Procedure. A trial consisted of a repeating sequence of four 300-msec images, diagrammatically indicated by $S \rightarrow M \rightarrow S^{\prime} \rightarrow$ $M$ and illustrated in Figure 1. The first and third images $\left(S, S^{\prime}\right)$ depicted either a crib or a workbench scene as described above. These two scenes either were identical (a no-change trial) or differed with regard to a single object (a change trial). The second and fourth images of the sequence $(M)$ depicted a full-screen colored noise mask. This mask was created by taking that trial's "empty" scene (i.e., the background without objects) and adding $100 \%$ uniform noise using the "add noise" filter supplied in Adobe Photoshop 4.0. The introduction of a noise mask in this paradigm served two functions. First, it disrupted any apparent motion percept that might accompany the sequential presentation of similar images; second, it removed the sudden visual transient that would otherwise make the detection of change between $S$ and $S^{\prime}$ trivial (Rensink et al., 1997). Figure 1 shows an example of a change trial involving the trumpet in $S$ and the baby bottle in $S^{\prime}$. The sequence of four images described above repeated until the trial was terminated by a buttonpress. The observer's task was to indicate the presence or absence of a change as quickly as possible without sacrificing accuracy. Judgments were indicated by two hand-held buttons, with the change response mapped to the button held in the dominant hand. Participants were allowed 25 practice trials to familiarize themselves with this task, and the observer initiated each trial by pressing a button. The experiment lasted approximately $1 \mathrm{~h}$, with the exact duration depending on the number of breaks requested by the participant. No reaction time (RT) or error feedback was provided.
Design. Each observer participated in 400 randomly interleaved change and no-change trials. The 200 change trials were divided into four conditions corresponding to the primary manipulations. The first manipulation varied the categorical relationship between the prechange object appearing in scene $S$ and the postchange object appearing in $S^{\prime}$. Changes could occur either within a given superordinate category, meaning that a tool would change with another tool or a toy with another toy, or across category, meaning that changes would occur between a toy and a tool. ${ }^{3}$ The display locations of the changing objects were predetermined to ensure that a change occurred equally often in all 18 of the allowable positions. The nonchanging objects on each change trial (the distractors) were then randomly assigned to 8 of the remaining 17 locations. The second change manipulation involved the orientation of the objects composing the target. All of the objects had their major axes rotated roughly $45^{\circ}$ clockwise or counterclockwise from vertical, meaning that the pre- and postchange objects either had a similar orientation (both were left-leaning or right-leaning) or a different orientation (one object leaning left and the other leaning right). Thus, across the two scene types (crib and workbench), there were 50 similar and 50 different orientation target pairs per category condition.

Figure 2 shows two representative change trials illustrating these category and orientation manipulations. Both examples depict a single change cycle, with the topmost image in each indicating the prechange scene and the bottommost image indicating the postchange scene. The left scenario shows a within-category change trial in which the changing objects have a similar orientation. The left-
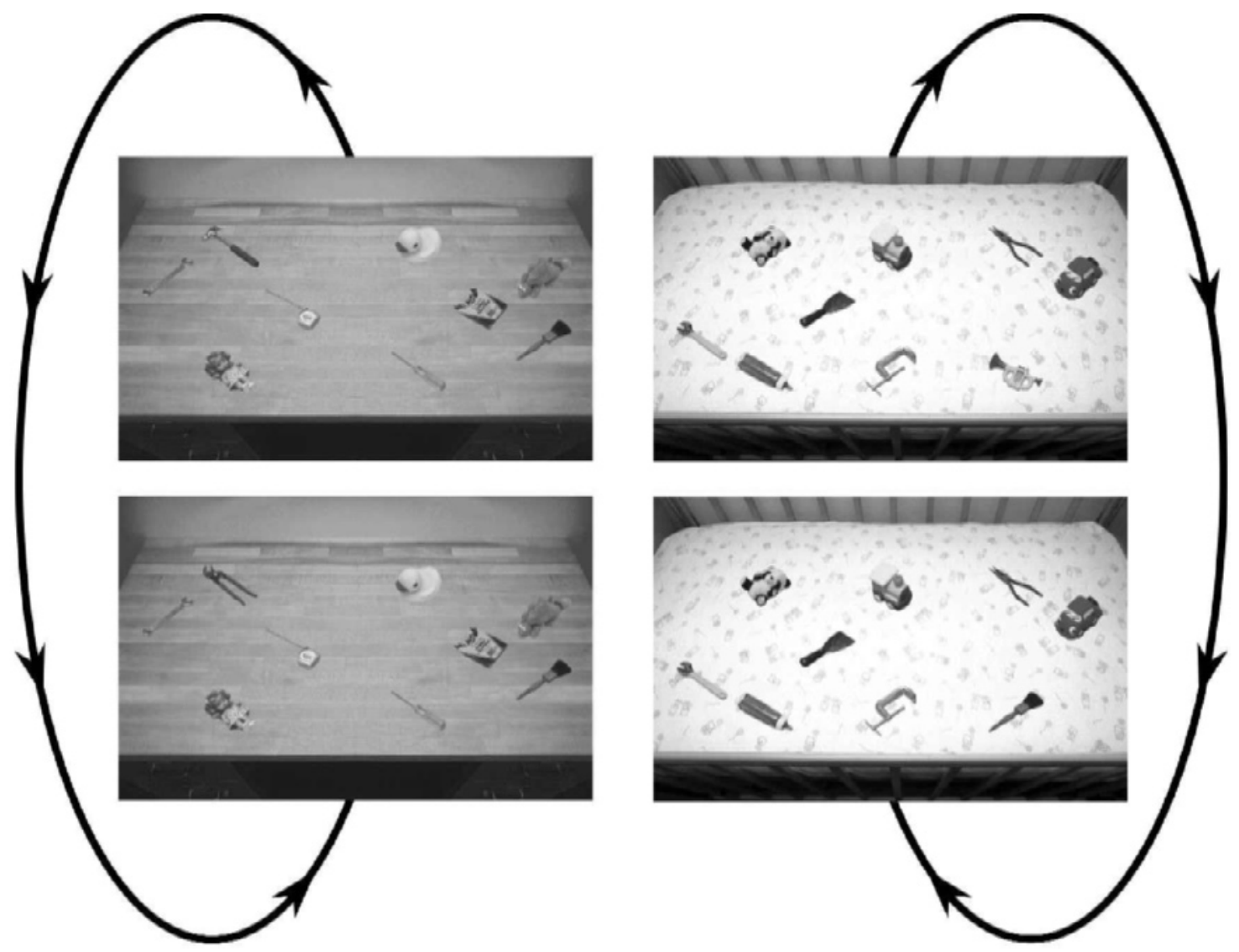

Figure 2. Two representative change trials from Experiment 1. The left scenario shows a within-category (tools) similar orientation (left-leaning) change between the hammer and channel-lock pliers. The right scenario shows an acrosscategory different orientation change between the left-leaning toy trumpet and the right-leaning paintbrush. Not shown in this figure are the within-category different orientation and the across-category similar orientation change conditions. 
leaning hammer in the prechange workbench scene changes with the left-leaning pliers in the postchange scene. The right scenario shows a case in which a change between the paintbrush and the trumpet violates both category and orientation, this time within the context of a crib scene. Notice from these examples that the background and distractors were identical between the pre- and the postchange scenes, and that four of the distractor objects were always inconsistent with the scene type. Regardless of the background (crib or workbench), half of the distractors would therefore be toys and the other half tools. Note also that the change always involved the substitution of one object with another (thereby not altering the configuration of display items), and that the substituted object would not have appeared elsewhere in either of the two scenes. ${ }^{4}$ As in the case of the change versus no-change manipulation, category and orientation change trials were randomly interleaved throughout the experiment.

No-change trials, not illustrated in Figure 2 and of generally less theoretical importance to this study, were nevertheless needed in order to encourage unbiased decision criteria and to provide a baseline against which change detection performance could be gauged. The 200 no-change trials were created by simply splitting apart the two scenes constituting the 50 within- and 50 across-category object change pairs, and presenting each of these scenes in an alternating fashion with itself.

\section{Results}

It was my expectation that changes violating category and orientation would generate larger change signals and would be easier to detect. It follows from this hypothesis that observers might also frequently miss changes in the within-category and similar-orientation trials because the change signal would be comparatively small in these conditions. Figure 3a indicates precisely these relationships. When the pre- and postchange objects had a similar orientation and came from the same category, observers responded incorrectly on $24.1 \%$ of the trials. Accuracy improved when these within-category changing objects differed in orientation, with the miss rate dropping to only $7.5 \%$. This effect of orientation similarity extended to the across-category data, where misses occurred on $7.1 \%$ of the similar-orientation trials but on only $2.1 \%$ of the trials violating object orientation. A two-way repeated- measures analysis of variance (ANOVA) revealed the expected main effects of orientation $[F(1,9)=40.83, p<$ $.001]$ and category $[F(1,9)=18.47, p=.002]$, as well as evidence for an interaction between these factors $[F(1,9)=$ $27.25, p=.001]$.

Identical patterns were observed for RTs (Figure 3b). Changes between categorically related objects having a similar orientation took the longest to detect, with performance again improving as the mismatches between the changing objects grew larger. Specifically, detection times for the different-orientation within- $(2,462 \mathrm{msec})$ and across-category $(2,129 \mathrm{msec})$ conditions were faster than their similar-orientation counterparts $(2,828 \mathrm{msec}$ and 2,286 msec, respectively). As in the case of manual errors, a repeated measures analysis of these RT differences yielded main effects of category $[F(1,9)=34.67$, $p<.001]$ and orientation $[F(1,9)=27.04, p=.001]$, as well as a significant interaction $[F(1,9)=5.62, p=.042]$. This consistency between the error and RT data argue against a performance tradeoff between accuracy and speed in this task. If observers delayed their within-category or similar-orientation responses in hopes of improving the accuracy of these judgments, then fewer errors should have been observed in these conditions. The fact that errors and RTs increased for both of these high-similarity conditions suggests that this tradeoff strategy was either not used or used unsuccessfully. A speed-accuracy tradeoff, however, did nicely characterize performance in the no-change trials, with observers apparently electing to maintain a low false alarm rate $(1.7 \%)$ by prolonging their search for the changing objects $(3,942 \mathrm{msec})$.

\section{Discussion}

The observed pattern of results is consistent with a very straightforward and intuitive axiom: the detection of change between two pseudo-realistic scenes varies with both the orientation and the categorical similarity of the changing objects. When the objects have a similar orientation or category, detection performance is quite a

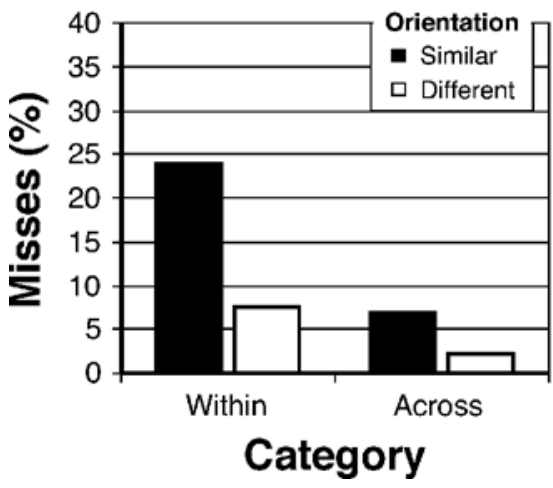

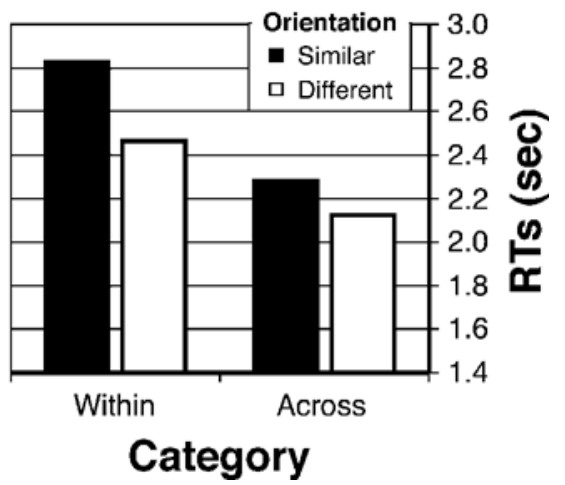

Figure 3. Buttonpress errors (left) and mean reaction times (right) for each of the category and orientation change conditions from Experiment 1. Note that both errors and RTs increased with the similarity between the changing objects. 
poor as measured by either error rates or RTs; when the changing objects are dissimilar, detection is faster and highly accurate. Both data patterns are also consistent with several recent studies reporting effects of similarity on change detection for a variety of featural dimensions (Scott-Brown et al., 2000; Scott-Brown \& Orbach, 1999; Williams \& Simons, 2000; Zelinsky, 1998, 1999, 2001a).

The observed orientation and category effects are difficult to reconcile with attention- and memory-based explanations of change detection failure that assume processing constraints imposed on items presented within a given scene. According to an attention-based explanation, objects central to the context of a scene (i.e., "centers of interest") are more easily detected when changed, because these objects are better attractors of attention and are therefore preferentially represented (Hollingworth \& Henderson, 2000; O’Regan, Deubel, Clark, \& Rensink, 2000; Rensink et al., 1997). Such an attention-based constraint, however, cannot easily explain the detection advantage for across-category and different-orientation changes in the present data. Both of the manipulated categories (tools and toys) and orientations (left- and rightleaning) were equally represented among the prechange objects, making the objects unremarkable with regard to these dimensions and rendering an explanation of the data based on preferential encoding implausible. A similar argument can be made against a memory-constrained detection process, at least one appealing to an object-based capacity limit on the number of items that can be encoded from a display. According to such a constraint, an observer may wish to encode many or all of the objects in a display, but is prevented from doing so when the number of display items exceed working memory capacity (Hayhoe, Bensinger, \& Ballard, 1998; Irwin, 1992, 1996; Irwin \& Andrews, 1996; Kahneman, Treisman, \& Gibbs, 1992; Pylyshyn \& Storm, 1988). The items represented in working memory are therefore limited to only a subset of the display items, thereby creating the potential for change detection failure. However, as in the case of an attention-based constraint, this explanation cannot easily account for the observed data. Because the manipulated categories and orientations were equally represented in the display, and violations were not defined until the appearance of the postchange scene, the subset of prechange items selected for working memory representation could not be biased to include objects from the across-category and differentorientation conditions. 5

If attention- and memory-based constraints were not limiting the information available from items in a display, what other factor might be responsible for the observed change detection performance? One possibility is that detection performance is limited, not by the operations encoding the items from a scene, but rather at the level of the operation comparing one scene with another. Several studies have suggested a potential link between change detection failures and the comparison operation (Rensink, 2000a; Scott-Brown et al., 2000; Simons, 2000;
Zelinsky, 1998, 1999, 2001a); but the possibility deserves further consideration, given its profound theoretical consequences. For example, a model positing performance limitations at the level of the comparison operation need not also hypothesize limits on serial encoding (an attentional constraint) or representational capacity (a memory constraint). ${ }^{6}$ In fact, even an unlimited-capacity parallel model could, in theory, generate change detection failures if the comparison operation was not successful in signaling a change. According to such a model, basic feature information might be represented in parallel for every object in the prechange display, and then compared, in parallel, with corresponding object representations in the postchange scene in order to generate a difference signal. The source of constraint in this model would be the representational overlap or similarity between the pre- and postchange patterns undergoing comparison. Framed in terms of the present data, detection may have been fast and accurate when the changing objects violated orientation or category because these manipulations decreased the representational overlap between the target patterns. However, when the changing objects shared the same superordinate category and had a similar orientation, a smaller difference signal would result from the comparison operation, thereby making the change detection task more difficult. Of course such an appeal to similarity as a source of constraint begs the questions of how the similarity relationships between real-world patterns can be quantified and used to drive change detection behavior. Experiment 2 addressed these topics by (1) framing an unlimited-capacity parallel model of change detection, (2) describing a method of constraining this model using the representational overlap of the patterns being compared, and then (3) examining how well this approach can explain the behavioral data from Experiment 1.

\section{EXPERIMENT 2 A Computational Model of Real-World Change Detection}

Despite offering a fairly straightforward account of the Experiment 1 data, visual similarity relationships are often left unspecified in change detection studies using realistic stimuli (see Simons et al., 2000, for a recent exception). One reason for this frequent omission is likely due to the difficulties associated with quantifying similarity relationships between real-world objects. Although similarity is a widely used and relatively accepted construct in the cognitive science community (Duncan \& Humphreys, 1989; Edelman, 1998; Gati \& Tversky, 1984; Goldstone, 1994b, 1994c; Raymond, Shapiro, \& Arnell, 1995; Tversky, 1977), saying that a behavior varies with similarity is a statement of questionable usefulness (Goodman, 1972; Murphy \& Medin, 1985; Watanabe, 1985; but see Medin, Goldstone, \& Gentner, 1993, for a more hopeful perspective). The problem is that the term is rather slippery and easily abused, and the application 
of the concept to real-world objects magnifies its potential for misuse. Whereas establishing visual similarity relationships between simple stimuli can be easily accomplished by varying some incontrovertible feature of the object, determining similarity relationships for realworld objects is far less trivial. A rubber ducky and a toy trumpet may both be predominantly yellow, but they also have very different shapes. How then would the similarity between these two objects compare to objects having a different color but a more similar shape?

Given the complexity of real-world similarity relationships, and the potential for biases and arbitrary criteria to creep into such estimates, theorists must be reciprocally explicit in their definitions of similarity and clear with regard to the aspect of similarity being addressed. One successful solution to this problem defines psychologically meaningful multidimensional spaces on the basis of observer similarity ratings or confusions and then sets decision criteria within these spaces to differentiate between models of behavior (Ashby \& Lee, 1991; Ashby \& Perrin, 1988; Nosofsky 1986, 1992). This approach is useful because it captures a wide range of similarity relationships between complex stimuli regardless of their level of abstraction, but it is not well suited to a targeted perceptual analysis in which only visual dimensions should be considered. For the purpose of the present discussion, I limit my definition of similarity to purely visual dimensions (e.g., color, orientation, and spatial scale), while recognizing that nonvisual dimensions might also affect the behavior in question (Goldstone, 1994a; Medin, Goldstone, \& Gentner, 1990; S. E. Palmer, 1978). To isolate this visual similarity component, I adopt a filter-based computational approach that decomposes a complex stimulus into multiple featural dimensions, making this method similar in spirit to many other approaches currently being used in the computational vision literature (Adini, Moses, \& Ullman, 1997; Amit \& Geman, 1997; Ballard, Hayhoe, Pook, \& Rao, 1997; Bricolo, Poggio, \& Logothetis, 1997; Edelman, 1993, 1998; Itti \& Koch, 2000; Lades et al., 1993).

\section{The BOLAR Representation}

Before information from real-world stimuli can be used to guide behavior, that information must first be extracted and represented. The job of extracting information from an image requires some sort of filtering mechanism. Filters can be constructed for a variety of featural dimensions, with the specific construction of the filter determining the type of information extracted from the scene. If a task requires quantifying the amount of a particular color or orientation or spatial scale (size) appearing in a scene, filters sensitive to all of these properties need to be constructed and applied to the image. Moreover, if multiple levels of featural information are desired within each of these dimensions (multiple orientations, multiple colors, etc.), the extraction of these features would also require dedicated filters. Filters also have a shape, which enables them to respond selectively to edges or bars or other forms of spatial structure in a pattern. This shape-selective property is particularly important for real-world representation, given the variability in spatial structure characteristic of realistic objects and scenes.

Filter-based models have been enormously successful in describing early visual processing, and it is not my goal at this time to apply each of these models to a change detection task or to advocate one of these models over another (Bell \& Sejnowski, 1997; Graham, 1992; Lades et al., 1993; Malik \& Perona, 1990; Rohaly, Ahumada, \& Watson, 1997; see Landy \& Movshon, 1991, for a review). ${ }^{7}$ Rather, my goal is to distill from these models a simplified and easily implemented approach tailored to the demands of a change detection task. Many models of early vision strive to fit data from neurophysiological recording or near-threshold psychophysics, but this level of specificity is probably unnecessary to a suprathreshold change detection task in which the stimuli are perceptually distinct real-world objects. More important to change detection is representational breadth, the coding of many different featural dimensions at multiple levels. Given the multiplicative relationship between featural dimension (e.g., orientation) and level (e.g., $60^{\circ}, 45^{\circ}$, etc.), and the need for representational breadth (many dimensions), it is easy to see how the number of filters needed to represent a realworld stimulus can quickly grow quite large. Such a high-dimensional representation would create a sort of featural signature for the patterns appearing in the change detection displays. There are two clear advantages to this approach. First, because a filter-based model can be applied to arbitrarily complex stimuli, both simple and complex scenes can be coded in terms of the same base featural primitives. Second, because each of these featural signatures exist in a relatively high-dimensional space, it would no longer be necessary to hand-pick the features to be compared in the change detection process. A change between a horizontal and a vertical bar will generate a response in the feature vector coding for "horizontal" and "vertical," and a change between a coffee cup and a plate of eggs will generate a response in whatever featural dimensions are specific to those patterns.

To achieve some measure of representational breadth, I currently use 108 Gaussian-derivative filters (GDFs) to code the visual features of a scene, with each filter being sensitive to a different spatial or chromatic property. As their name implies, the mathematical functions underlying GDFs are obtained by differentiating a three-dimensional Gaussian. The 108 GDFs used in this study can be broadly classified into three filter types, with each type corresponding to one of the first three derivatives of the Gaussian function. Recall that Gaussian differentiation yields as derivatives oriented functions numbering one more than the order of the derivation. A first-order derivation produces two directional (oriented) derivatives, a secondorder yields three, and a third-order yields four. These 9 oriented functions were used to represent or filter orientation information in the change detection stimuli. 
Specifically, first-order filter responses were collected at $0^{\circ}$ and $90^{\circ}$ orientations; second-order filters were constructed for $0^{\circ}, 60^{\circ}$, and $120^{\circ}$ orientations; and thirdorder responses were collected at $0^{\circ}, 45^{\circ}, 90^{\circ}$, and $120^{\circ}$. Figure 4 illustrates a GDF for each of these three orders for a given orientation. Additional details regarding the selection of these particular orientations is provided in Appendix A and in Zelinsky (1998).

This set of nine oriented GDFs is repeated at four octave-separated spatial scales $(3 \times 3,7 \times 7,15 \times 15$, and $31 \times 31$ pixels), accounting for 36 of the 108 filters used in the current representation. The term scale in this context can be understood as meaning the size of a filter's "receptive field." Larger scale filters will "see" or acquire information over a relatively large region of the image, thereby enabling them to efficiently extract low spatial frequency patterns from a scene (e.g., the overall shape of an object). Small-scale filters will acquire information over a comparatively narrow region of the image, making them well suited to extract high spatial frequency patterns (e.g., the fine visual structure corresponding to object parts). Using filters of varying scales, the current representation therefore performs a coarse spatial frequency analysis of the change detection stimuli.

These 36 multiscale oriented GDFs are in turn repeated for three color/intensity channels, thereby accounting for all 108 of the filters used in the representation. The first 36 filters perform an achromatic analysis of the scene. Prior to any filtering operations, the red, green, and blue pixel values of the image are simply averaged to produce a grayscale scene. The remaining two channels are indeed chromatic-more specifically, they are color-opponent much like the human visual system. The second channel, Filters $37-72$ in the representation, operates on a red-green transformation of the image (meaning that the green pixel values are subtracted from the red, then normalized between 0 and 255). Similarly, the third channel, Filters 73-108, operates on a blue-yellow image transformation, with yellow being the average of the red and green pixel intensities. To summarize, this 108-dimensional filter-based representation can be evenly divided into three color/luminance channels, with each channel in turn being divided into four spatial scales and finally into nine oriented GDFs at each scale.

Figure 5a shows the responses from all 108 GDFs following their application to the trumpet image. The $x$-axis indicates the type of filter generating the response, with each bar corresponding to a single filter responding in proportion to the presence of specific spatiochromatic properties in the image. The $y$-axis indicates the magnitude of these filter responses. To obtain these responses, each of the 108 GDFs was centered over the midpoint of the trumpet image (i.e., the midpoint of the filter was aligned with the midpoint of the image) and then convolved with the portion of the image "covered" by the filter (i.e., a $3 \times 3$ filter would operate on only the 9 pixels surrounding the image midpoint, whereas a $31 \times 31$ filter would analyze a larger 961-pixel region of the image). The process of convolution entails (1) taking the pixel intensity for every image point under the filter, (2) multiplying this intensity by the corresponding filter value (ranging from -1 to 1 ), and (3) summing over the filter extent. The result of this operation yields a single number corresponding to the filter's response (see Appendix B for a more concrete example of this process). The specific pattern of responses appearing in Figure 5a illustrates why a filter-based approach is well suited to real-world representation. This pattern represents only one of an astronomically large number of potential patterns that can be generated by varying the responses from 108 filters. A very different pattern might emerge if filter responses were collected from a point even a few pixels away. This 108-dimensional pattern therefore describes a unique featural signature identifying a particular region in an image, a property that can be exploited to generate a change signal.

I group these 108 filter responses into a larger functional unit of visual analysis that I have termed a BOLAR (bank of local analyzer responses) vector. A BOLAR is the specific 108 responses obtained by convolving a bank of spatially coincident GDFs with an image, with the featural signature appearing in Figures $5 \mathrm{a}$ and $5 \mathrm{~b}$
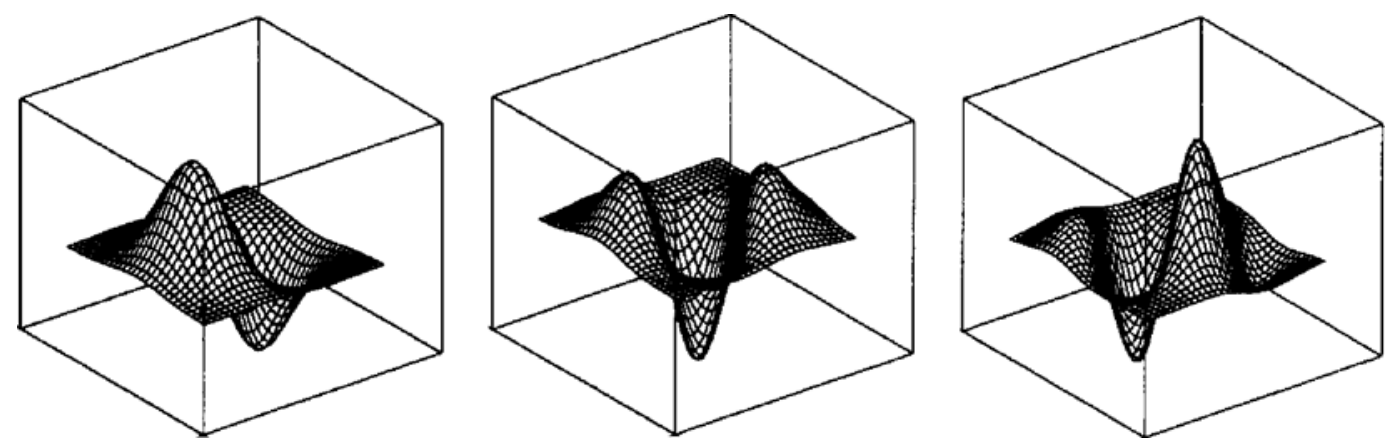

Figure 4. The shapes of the first- (left), second- (middle), and third- (right) order Gaussian-derivative filters. Note that filter scale is not specified in this figure, and that the three depicted filter orientations $\left(9^{\circ}, 120^{\circ}, 120^{\circ}\right)$ were chosen to best illustrate the filter's shape. 


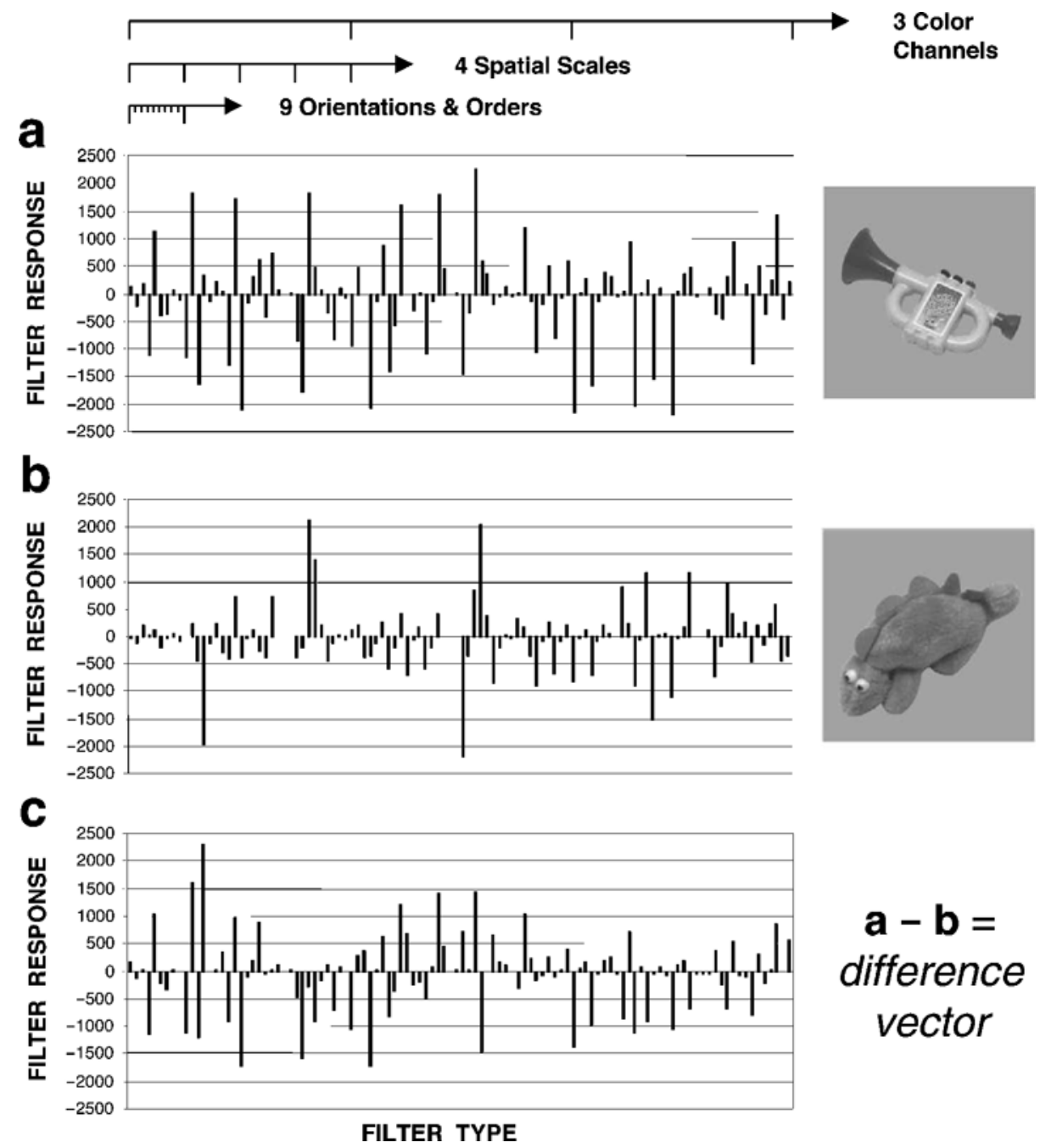

Figure 5. The 108 filter responses comprising a BOLAR vector. Representative responses are shown for two BOLAR vectors, one centered on the midpoint of the toy trumpet image (a) and the other centered on the dinosaur doll image (b). The midpoint difference vector (c) reflects the pairwise subtraction of vector $b$ from $a$.

being two examples of BOLAR vectors (both computed for the image midpoints). A BOLAR can therefore be conceptualized as a collection of filters dedicated to the analysis of a localized patch of visual space, similar to the hypercolumn organization of striate cortex described by Hubel and Wiesel (1962). Like the hypercolumn idea, BOLARs represent a fairly low-level visual description of the world. Patterns described at the BOLAR level are not yet "objects" in any high-level meaning of the word. They need not have names or any abstract functional properties associated with them, nor do they even have to be visually segmented from their backgrounds. However, BOLARs break from the hypercolumn analogy in one key respect. In order to be a useful representational scheme for change detection, BOLARs have to exist in a scenebased coordinate system rather than the retinotopic sys- tem characteristic of V1, potentially localizing them in parietal cortex (Zipser \& Anderson, 1988). Once a BOLAR is computed for a location in space, it must remain attached to that location regardless of observer motion and gaze shifts. This scene-based property therefore makes BOLARs similar to FINSTs (Pylyshyn, 1989; Trick \& Pylyshyn, 1993) and object files (Kahneman \& Treisman, 1984; Kahneman et al., 1992), but rather than tracking objects moving through space, a BOLAR tracks a pattern in space over eye, head, and body movement.

\section{Quantifying Visual Similarity Using BOLAR Representations}

The behavioral data from Experiment 1 suggested an inverse relationship between change detection performance and the similarity of the changing objects. The 
more similar the changing objects, whether in terms of orientation or of category, the more difficult the change detection task. BOLAR representations, by providing a means of quantifying visual similarity, allow a computational treatment of this relationship. In order to quantify the visual similarity (or dissimilarity) between any two objects undergoing change in Experiment 1, I first computed BOLAR vectors for every pair of corresponding points in the two images, then found the normalized Euclidean distance for each of these vector pairs using the equation:

$$
E=\frac{1}{n} \sqrt{\sum_{k=1}^{n}\left[\boldsymbol{b}_{k}-\boldsymbol{b}_{k}^{\prime}\right]^{2}},
$$

where $n$ is the dimensionality of the BOLAR representation and $\boldsymbol{b}$ and $\boldsymbol{b}^{\prime}$ the two BOLAR vectors being compared. Figure 5 helps to illustrate this operation. The BOLAR vectors shown in Figures $5 \mathrm{a}$ and $5 \mathrm{~b}$ were derived for corresponding points (in this case, the midpoints) in the trumpet and dinosaur images. The vector shown in Figure 5c was obtained by simply subtracting the $5 \mathrm{~b}$ vector from the $5 \mathrm{a}$ vector. This difference vector was then reduced to a single nonnegative number (the Euclidean distance) by squaring and summing each of its 108 difference signals and then taking the square root of this value.

Performing the above-described operation for every corresponding point in the two images, and then normalizing these Euclidean distances between the range of 0-255 for visualization, results in the creation of a difference map. Figure 6 shows two such difference maps, together with the image pairs used in their generation. A difference map represents the BOLAR-derived visual dissimilarity between any two patterns. As a result of the normalization process, dissimilarity in these maps is represented by intensity, with brighter points indicating a greater difference signal. This dissimilarity is expressed in two ways. First, notice that there are more bright regions in the left map than in the right, with these regions representing evidence of dissimilarity or change. This greater expression of dissimilarity between the leftmost objects is partially related to the orientation mismatch between the panda bear and crescent wrench patterns. Objects having different orientations typically generate more regions of change than in similarly oriented objects, and this relationship is captured by the difference map. A second expression of dissimilarity in these images can be seen if we compare the intensities of the change regions. The pronounced region of change in the left difference map (where the head of the wrench meets the leg of the bear) is $30 \%$ brighter than the largest difference signal appearing in the right map. This dual coding of visual similarity, in terms of both degree (intensity) and distribution (regions in the image), makes the difference map a potentially useful means of visualizing the signal underlying change detection.

It is possible to further reduce the information on a difference map to obtain a single number representing the visual similarity between two patterns. Rather than plotting each of the pair-wise Euclidean distances in the form of a map, a single similarity estimate was obtained by summing these difference signals and converting them to a similarity metric. Intuitively, this method is equivalent to comparing the overall amount of white in two difference maps when judging whether one pair of objects is more visually similar than another. Using this method, I computed the visual similarity for every combination
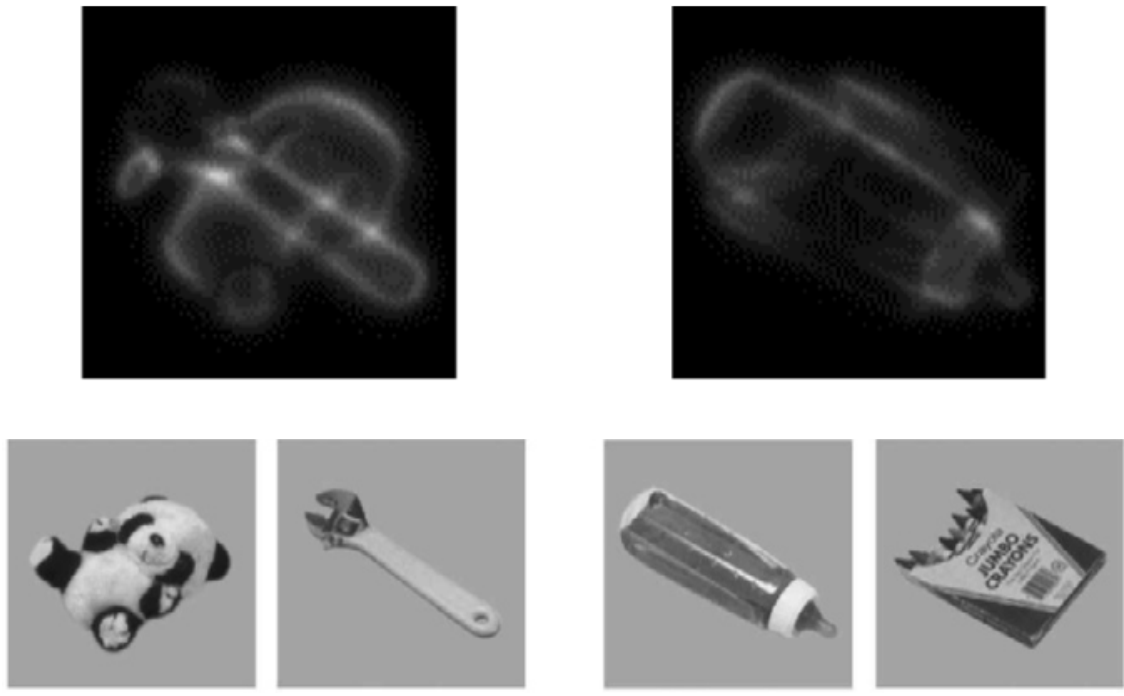

Figure 6. Two difference maps (top), each shown with the objects used in the difference computation (bottom). Note that the object pair violating orientation and category (the panda bear and crescent wrench) produces a brighter and more distributed region of change than do the more similar baby bottle and crayon objects. 
of pre- and postchange objects used in Experiment 1. Figures 7 and 8 show these BOLAR-derived similarity estimates. The objects in the left column of each figure were compared with the objects in the top and bottom rows. Similarity estimates are shown in each cell of the matrix, with the value above the diagonal indicating the similarity between a left column object and the corresponding item in the upper row and the value below the diagonal indicating the similarity relationship for the lower object. In order to make meaningful similarity comparisons between different object pairs, the summed difference signal for each object pair was normalized relative to the most dissimilar object pair in the set. This involved (1) finding the object pair having the greatest summed difference signal, and then (2) dividing the summed difference signal for each object pair by this maximum. This dissimilarity measure was then converted to similarity by subtracting the obtained value from 1 . The maximally dissimilar ducky and clamp objects in Figure 8 therefore have a similarity estimate of
0, whereas the box wrench and paintbrush objects in Figure 7 have the highest estimated similarity of .89 (a similarity of 1.0 would be obtained if an object were compared with itself). The location of these values with respect to the diagonal represents whether the comparison was within or across categories. Entries above the diagonals indicate within-category comparisons, with similarity estimates for the tools appearing in Figure 7 and estimates for the crib objects appearing in Figure 8. Entries below the diagonals in each figure, separated here purely as an illustrative convenience, indicate acrosscategory object similarities.

Inspecting these matrices, one can easily find cases in which the BOLAR similarity estimates map onto a subjective impression of object similarity. For example, the estimated similarity between the tape measure and the C-clamp in Figure 7 is .22, a relatively low value reflecting the very different shape and orientation of these two objects. However, the hammer and the screwdriver in the same figure have a much higher BOLAR similarity of
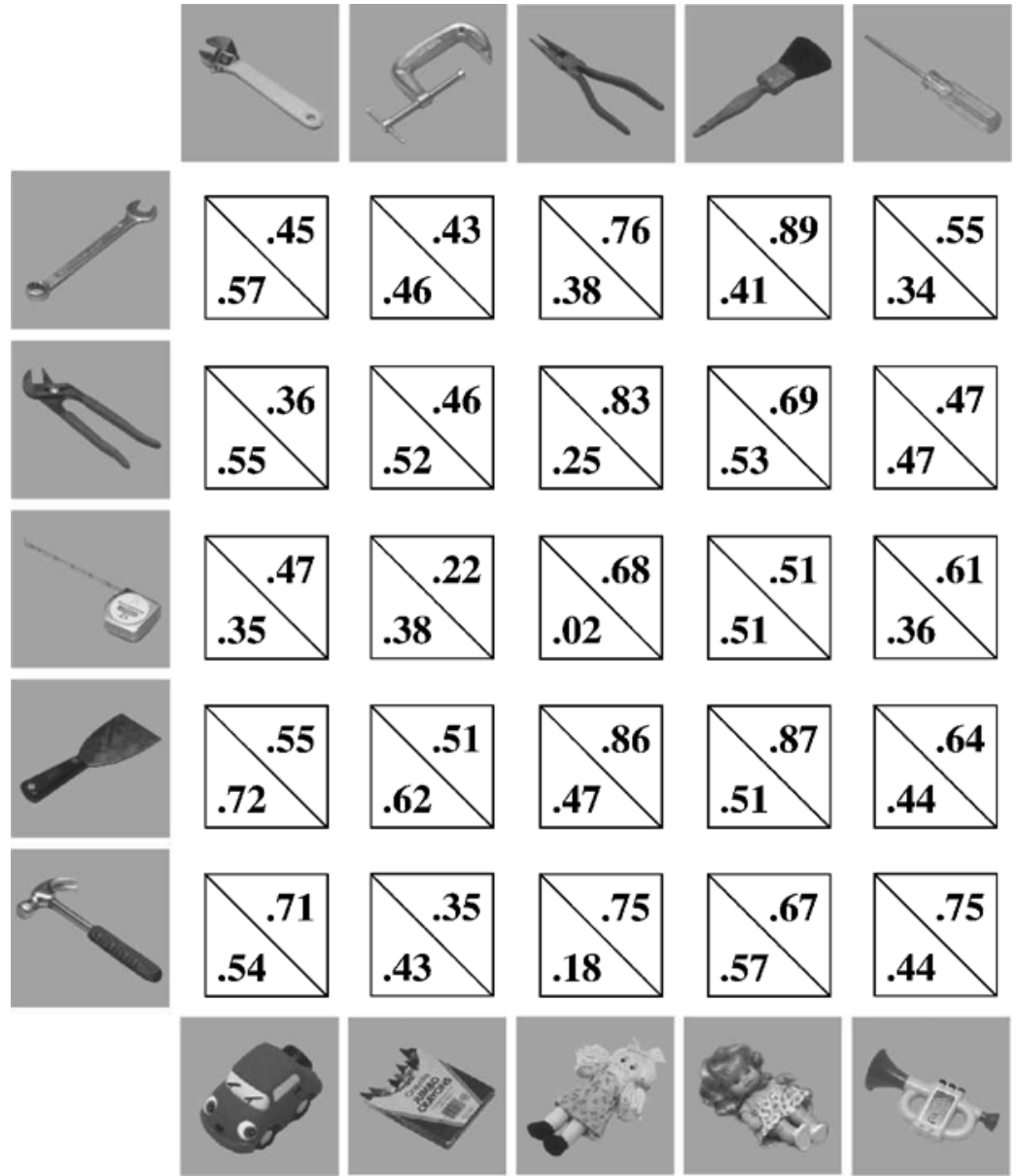

Figure 7. Object similarities as estimated by BOLAR representation. All of the objects paired in this matrix were "changing objects" in Experiment 1. Note that values above the diagonals represent within-category comparisons between the workbench objects, whereas values below the diagonals represent across-category comparisons between toys and tools. 

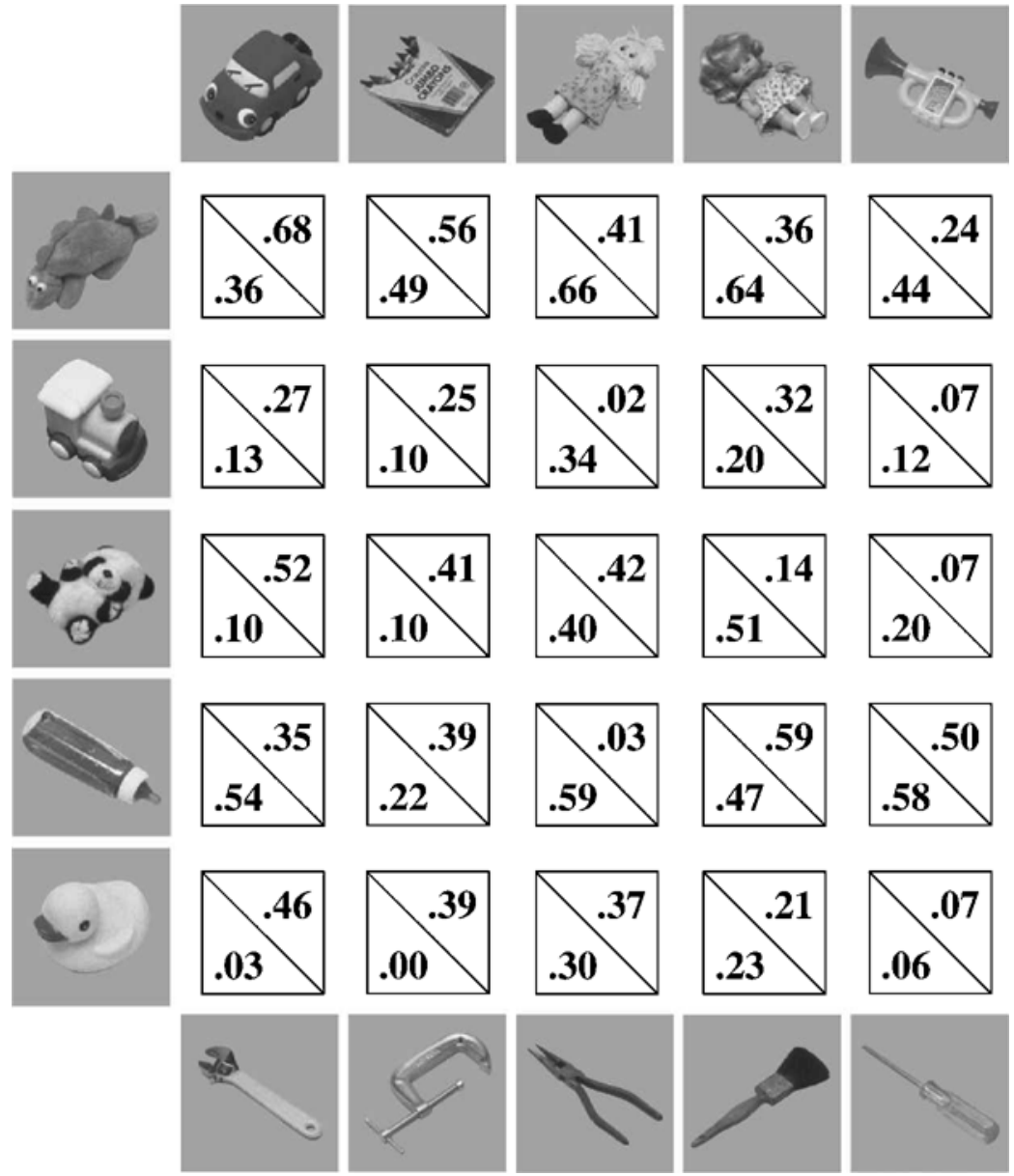

Figure 8. More BOLAR-based object similarity estimates. The within-category comparisons in this figure (values above the diagonal) indicate visual similarity between the crib objects. The below-diagonal values are a continuation of the acrosscategory comparisons left incomplete in Figure 7.

.75. Subjectively these objects might also appear more similar, owing to the fact that they both have a similar orientation and elongated shape, as well as a brownishyellow hue (not visible in the illustration).

Because such subjective estimates of purely visual similarity can be misleading, I segregated the 100 values appearing in Figures 7 and 8 into the same change conditions that were used in Experiment 1. These mean similarity data are shown in Figure 9. When the changing objects had a like orientation and came from the same category, similarity estimates based on BOLAR representation were quite high, averaging $.53( \pm .01 S E M)$. However, when these categorically related changing objects had different orientations, estimated similarity dropped sharply to only $.39( \pm .02)$. An identical pattern was found for objects coming from different superordinate categories. The average similarity for objects in the across-category similar orientation condition was .42 $( \pm .01)$, and the lowest similarity of $.33( \pm .02)$ was found when the change violated both category and orientation.
This decreasing trend in the similarity estimates is qualitatively similar to the patterns of human errors and RTs shown in Figure 3, suggesting that a difference signal based on the visual comparison of two objects may be used in change detection behavior.

In order to better quantify the relationship between these behavioral and computational measures, I correlated the BOLAR-derived similarity estimates with the RT data from Experiment 1. The obtained mean correlation for observers was .669, with the correlation for each observer being significantly greater than zero $[F(1,98) \geq 22.88, p \leq$ $.001]$. To determine the $\mathrm{RT} \times$ similarity correlations irrespective of the category and orientation manipulations, I segregated the data into the four Experiment 1 conditions and conducted separate analyses. Within-category correlations were .694 and .704 for the similar and different orientation conditions, respectively. Correlations of .633 and .667 were found for the corresponding across-category conditions. All of these correlations were reliable by observer $[F(1,23) \geq 4.90, p \leq .037]$. To summarize these 


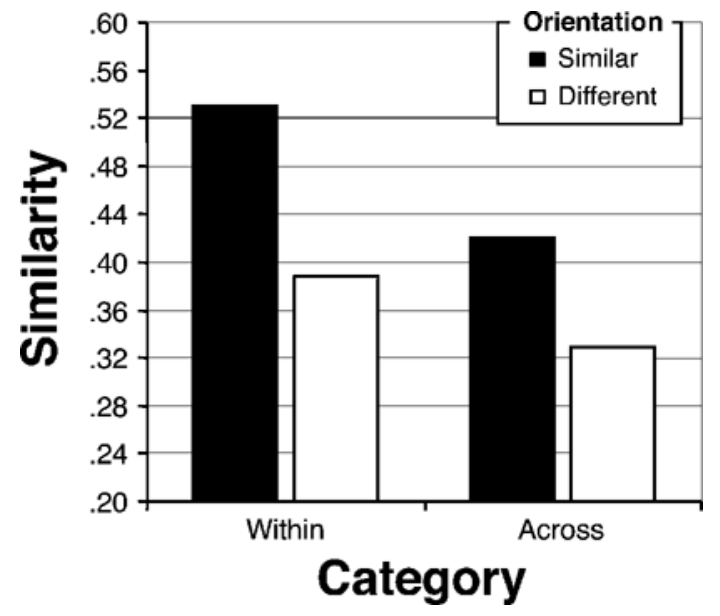

Figure 9. Mean similarity estimates for all of the object change pairs used in Experiment 1 (Figures 7 and 8), grouped by change condition. Note that changes violating category and orientation produce smaller filter-based similarity estimates- the same trend characterizing the patterns of manual errors and RTs appearing in Figure 3.

analyses, the time taken to detect an object change varied directly with the visual similarity of the changing objects, with the longest RTs corresponding to the objects estimated to be visually most similar.

Of particular interest in Figure 9 is the main effect of category captured by the BOLAR model. This difference suggests that objects coming from the same superordinate category tend to be more visually self-similar $(M=$ $.46 ; S E M=.01)$ than objects coming from different superordinate categories $(M=.37 ; S E M=.01)$ —at least for the objects and categories used in the present study. One intriguing implication of this finding is that the effect of category reported in Experiment 1 may be explained, in part, purely by visual similarity. If the tool stimuli were visually more similar to the other tools, and the toys were visually more similar to the other toys, then an acrosscategory object change would, on average, generate a larger difference signal than a within-category change. A reasonable question to ask then is whether any additional advantage is gained by the nonvisual categorical nature of the object changes? To examine this issue, I first conducted a multiple linear regression using the mean RT data from Experiment 1 as the dependent variable and the BOLAR similarity and category factors as independent variables. This analysis yielded an adjusted $R^{2}$ of .735. Next, I partialled out of this correlation the contribution of visual similarity to determine what portion of the remaining variability could be explained by a pure effect of category. Controlling for BOLAR similarity, the correlation between category and RT dropped to .420 , meaning that only $18 \%$ of the variability in these change detection responses can be attributed to the category manipulation irrespective of visual similarity. Returning to the question motivating this analysis, although superordinate category violations may have influenced performance in this task for reasons unrelated to visual similarity, it is also true that category-related visual similarity relationships were the principle contributor to the within- versus across-category differences reported in Experiment 1. Specifically, $76 \%$ of the change detection variability resulting from the category manipulation could be explained by purely visual factors. Researchers manipulating categorical relationships between visually complex objects may therefore want to consider visual factors when interpreting their data.

Two implications follow from these relationships between human behavior and the BOLAR model. First, they suggest that the similarity estimates generated by the BOLAR model have psychological validity. BOLARestimated visual similarity was able to account for up to $49 \%$ of the variability in the RT data. The degree of psychological validity offered by these correlations is a matter of interpretation. Certainly BOLARs do not account for all (or even most) of change detection variability, which is not surprising, given the host of other factors that might influence this behavior. However, the visual similarity between the patterns undergoing comparison is a significant determiner of change detection performance, and BOLARs can be a useful means of quantifying this component. Second, these relationships suggest that it may be possible to use BOLAR-derived visual similarity estimates to constrain an unlimited-capacity parallel model of change detection. The following section briefly outlines how such a model might work.

\section{Toward Implementing a BOLAR Model of Change Detection}

In the preceding discussion of similarity estimates, most of the tools needed for implementing an unlimitedcapacity parallel model of change detection have been assembled. Figure 10 shows a representative change detection trial in which the rag doll in the top scene changes with the tape measure in the middle scene. Each of these images was $256 \times 192$ pixels in dimension, and I again computed BOLAR vectors for each point in these images and plotted their normalized Euclidean distances as a difference map (Figure 10, bottom). Most of the difference map is black, because the two scenes are identical except for a relatively small localized area surrounding the changing objects. As for the area in which the change actually exists, it appears as a bright region on this difference map.

By quantitatively indicating areas of difference between two changing scenes, the BOLAR-derived difference image can be thought of as a generic form of saliency map (Itti \& Koch, 2000; Koch \& Ullman, 1985), with saliency here defined as the expression of change. Detecting change between these scenes can be accomplished by the equally generic process of setting a detection threshold and then checking to see whether points on the saliency map exceed this value. The simplicity of this process, however, begs an obvious question: Given 


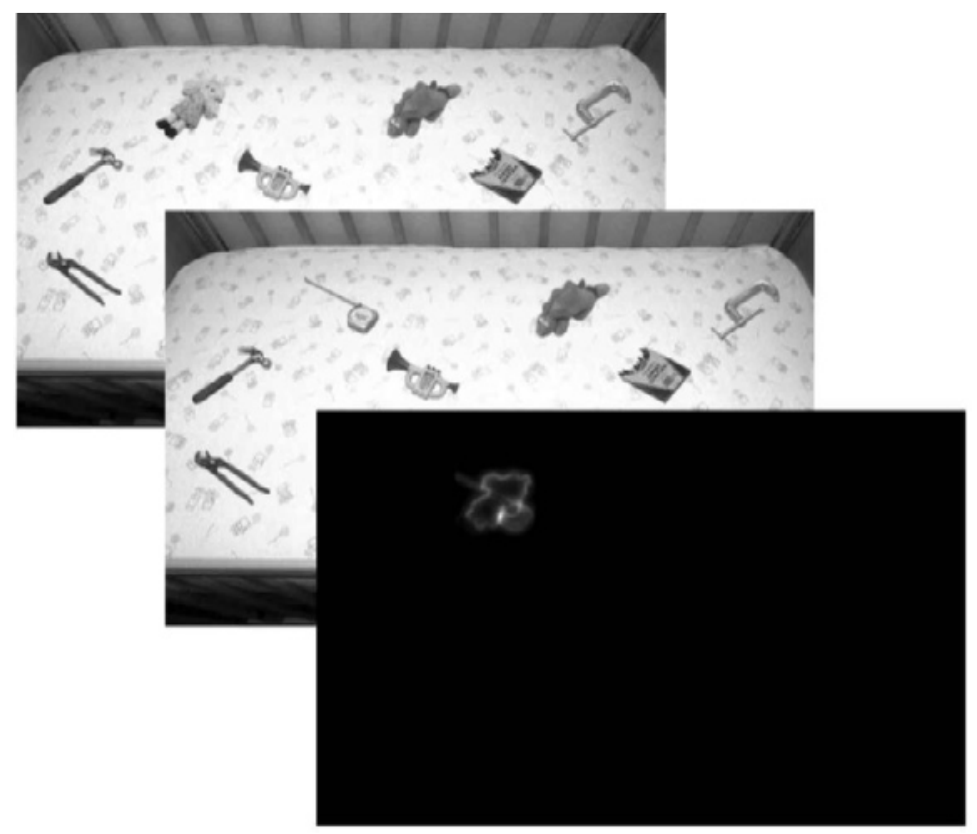

Figure 10. A BOLAR Euclidean distance is plotted for every corresponding point in two scenes comprising a representative change trial. Intensity on this "difference map" represents visual dissimilarity between the scenes, thereby indicating the presence of change.

the type of clear difference signal evident in Figure 10, why would any object change between two scenes not be immediately detected against the black no-change background? One answer to this reasonable question is that the background is probably seldom pure black. The difference map shown in Figure 10 is highly idealized, containing no noise that might mask the detection of change. It is well accepted, however, that internal representations in biological systems are inherently noisy (Graham, Kramer, \& Yager, 1987; Green \& Swets, 1966; Lu \& Dosher, 1999). Such noise would show up as points of change on a less idealized difference map, thereby undermining a simplistic threshold-based detection process and requiring more sophisticated methods of isolating the change signal. If one also allows for the possibility that new visual stimulation occurring during the ISI separating the change scenes might interfere with the retrieval of prechange information (i.e., a form of memory constraint), this factor too could be a source of considerable noise. ${ }^{8}$ The effect of noise sources on the detection process would be to make a change between two dissimilar objects (e.g., the panda and crescent wrench) easier to detect than a change between objects having a greater visual similarity (e.g., the baby bottle and the crayons).

\section{GENERAL DISCUSSION}

The present study advances change detection theory in three important respects. First, I have demonstrated a clear relationship between the visual similarity of the ob- jects undergoing change and change detection behavior. Although previous studies have recognized the potential for visual similarity to affect change detection performance, a systematic exploration of this constraint has been hampered by the inherent challenges in quantifying similarity relationships for real-world objects. Moreover, in the few studies that have responded to this challenge, these relationships were coded for only a small number of hand-picked featural dimensions. Not surprisingly, such a hit-or-miss strategy of choosing features has produced an unclear picture of how visual similarity contributes to change detection behavior, with some studies finding an effect of similarity (Williams \& Simons, 2000; Zelinsky, 1998, 1999, 2001 a) and others arguing against its importance (O'Regan et al., 2000; Rensink et al., 1997; Simons et al., 2000). Using the BOLAR representational framework developed for this study, I found that changes between visually less similar objects were easier to detect. It is unclear why an effect of visual similarity was observed in the present study, whereas Simons et al. found no evidence for such a relationship. Although the radically different methods used in these two studies make it difficult to pinpoint a specific reason for this discrepancy, one possible explanation may involve the coding of edge information in the change stimuli. The BOLAR model was sensitive to this edge information; the methods used by Simons et al. were not. Indeed, as described in Appendix B, even a filter-based model would be insensitive to change if spatial structure was removed from the stimulus. Whatever 
the actual source of the discrepancy, it appears that the BOLAR framework is the more sensitive measure for estimating visual similarity between complex changing patterns. The reason for this increased sensitivity is a straightforward by-product of the high-dimensionality of the BOLAR representation. Because the BOLAR model quantifies changes in terms of 108 featural properties, it is relatively unlikely that an effect of visual similarity will be missed as a result of the change-relevant featural dimensions' not being among those hand-selected for analysis.

The second contribution of the present study to change detection theory involves the general method used to quantify these visual similarity relationships. Despite the prominent use of realistic stimuli in the change detection literature, no computational model of change detection has been able to accommodate these stimuli. Consequently, theories of change detection have tended to be largely descriptive in nature, lacking the detail needed for one to properly evaluate their assumptions. The present work overcomes this obstacle to theoretical development by introducing an explicit computational framework for representing visually complex real-world objects. I purposefully designed the BOLAR model using fairly generic representational assumptions and made it relatively easy to implement, with the hope that researchers interested in rigorously describing their real-world stimuli might find value in the approach. Furthermore, I used common image processing techniques in building this model so that it can be applied to arbitrarily complex stimuli and therefore appeal to a wider range of researchers. It is immaterial to GDFs whether the represented patterns are simple shapes or fully realistic scenes. Regardless of their visual complexity, once these stimuli are decomposed into their constituent visual features, it becomes possible to directly compare the strength of their difference signals and to make meaningful inferences regarding their visual detectability in a change detection task. Such a "common language" is a necessary prerequisite for theory development, allowing performance constraints introduced by stimulus representation to be isolated and evaluated independently of higher level constraints imposed by processes acting on these representations.

The third theoretical contribution of this study is more speculative than the other two and involves pinpointing the source of constraint responsible for change detection failures. Three sources of potential constraint were discussed in the introduction. The first two, those involving attention and memory limitations, are commonly invoked in change detection studies to explain detection failures. The present investigation has focused instead on a third constraint, one involving the similarity between the pre- and postchange objects.

The BOLAR framework makes no appeal to attentionand memory-based constraints, and as such it can be considered an unlimited-capacity parallel model of change detection constrained by the similarity relationships between the changing objects. By highlighting the potential for a similarity-based constraint to explain change detection performance, the BOLAR model questions the need to invoke attentional and memory constraints to explain this. However, two points should be considered before adopting a similarity-constrained explanation for change detection. First, the present study has provided no direct evidence in support of an unlimitedcapacity parallel model, nor has it explicitly ruled out the influence of attentional or memory limitations. In this sense, the BOLAR model should be seen merely as an existence proof that a purely similarity-constrained model can generate change detection behavior; there is no reason at this time to believe that this is how observers actually go about performing a change detection task. Second, several well-reasoned arguments have been levied against the sort of unlimited-capacity parallel model that is represented by the BOLAR framework. For example, Rensink and colleagues (Rensink et al., 1997) reasoned that if change detection involved a comparison between two high-capacity visual buffers, performance should be fast, accurate, and largely automatic. Failing to find evidence for any of these patterns of response, they rejected the notion of an unlimited-capacity parallel model and instead explained their data in terms of an attentional limitation.

Because the argument offered by Rensink et al. (1997) is a fairly representative critique of an unlimited-capacity parallel model of change detection, it may be worthwhile to examine that argument in a bit more detail. Specifically, three aspects of their interpretation deserve further comment given the present findings. First, it is not the case that an unlimited-capacity parallel model implies perfect change detection performance. Even a model free of attentional and memory constraints might produce responses proportional to the magnitude of the change signal. One likely source of this graded change signal is the visual similarity between the patterns undergoing change. As the BOLAR model demonstrates, these visual similarity relationships can account for a significant portion of the variability in change detection performance-implying that a model constrained by these similarity relationships would likely do a reasonable job of describing change detection behavior. Second, although it is true that Rensink et al.'s argument against an unlimited-capacity model correctly rebukes the noise-free detection scenario illustrated in Figure 10, this argument requires modification before it can be applied to more realistic detection events allowing for noisy internal representation. Although the effects of visual noise have not yet been systematically studied in the context of a fully implemented BOLAR model, it should be fairly clear that even representation-intensive BOLAR vectors, if degraded by noise, will result in change detection difficulty in proportion to the degree of degradation. Third, the decision to adopt an attention-constrained theory of change detection over a similarity-constrained theory should be carefully considered and properly justified. An appeal to attentional selection as a cause of change detection failure, while sounding very informative, is tantamount to invoking the homunculus. In order 
for this to be a useful explanation, theorists must specify why attention should be preferentially directed to some objects but not others (see Scholl, 2000, and Zelinsky, 1998 , for further discussion of this point). Rensink et al. (1997) grappled with this question by suggesting that attention is drawn to the centers-of-interest in a scene; but note that calling something a center of interest essentially just redefines an object of attention and adds little to our understanding of why the object became an attentional attractor. Several other studies have targeted more specific perceptual or cognitive factors responsible for attentional allocation in a change detection task (Scholl, 2000; Wallis \& Bülthoff, 2000; Werner \& Thies, 2000). For example, Levin and Simons (1997) concluded that attention would likely be directed to a central figure in a scene, particularly if that figure was a person and the person was in motion. Note, however, that these studies fall short of establishing a causal link between attention and change detection, and many in fact suffer from an unsettling degree of circularity: Change detection is theorized to benefit from attention to a changed object, with proof of an object receiving this attentional allocation being improved change detection performance. Not surprisingly, appeals to attentional constraint as an explanation of change detection make a deceptively small theoretical contribution to our understanding of this behavior and will likely continue to do so until researchers explicitly address the representational substrate of the changing objects and the selection processes acting on these representations.

Does the similarity-based BOLAR model provide a better framework for integrating an attention process with a change detection task? By signaling the presence of change on a filter-derived difference map, the BOLAR model may be interpreted as generating the signal that directs attention to regions of high activation on such a map, thereby grounding the contribution of attention in the context of a computationally rigorous representational framework. However, even with this reconceptualization, attention remains a rather impotent theoretical construct. Attentional direction in this scenario is determined entirely by the activation map, with the real work therefore being accomplished by the change signal generating this activation. If it is the change signal that is used to guide attention to the target patterns, then a more parsimonious model might simply remove attention from the equation and base the detection judgment solely on the comparison operation signaling the change. Because the BOLAR model already explicitly describes the change signal, invoking the concept of attention adds little to the explanatory power of the theory, introducing instead an unnecessary level of complexity to the description of change detection behavior.

A theory should be evaluated, not only by how well it can explain the existing data, but also by the types and numbers of parameters used in that explanation. Theories of change detection relying on attention or memory constraints need to be particularly sensitive to the prob- lem of over-parameterization. Just as the assumption of multiple fixed-capacity resource pools overparameterized attention theory in the 1970s (see Allport, 1980, and Logan, 1997, for thoughtful reviews of this topic), the potential for memory or attentional constraints to exist during any or all of the encoding, retrieval, and comparison operations required by a change detection task threatens to create an unpleasant theoretical soup of free parameters. Such a soup is poison to a theory, rendering even the most insightful of explanations weak and useless as the number of parameters approach the number of data points requiring description. The BOLAR model of change detection has no free parameters. The model's behavior is determined completely by the similarityconstrained change signal resulting from the operation comparing the pre- and postchange scenes. This absence of free parameters is a bit of a double-edged sword. On the one hand, it all but guarantees that the BOLAR model will be limited in the amount of change detection variability that it can explain, perhaps accounting well for effects of visual similarity on detection performance but neglecting higher level factors that undoubtedly also affect this behavior. On the other hand, the fact that the BOLAR model assumes no memory or attentional bottlenecks makes it a useful baseline against which more constrained models of change detection can be evaluated. If it can be demonstrated that (1) a pattern of change detection data cannot be described by an unlimited-capacity parallel model, and that (2) the data can be explained by the addition of a memory or attentional constraint targeted to a specific operation in the detection process, then the inclusion of this parameter can be embraced with an added degree of confidence that it is making a meaningful contribution to change detection theory.

\section{CONCLUSION}

Change detection is a composite behavior consisting of both high- and low-level factors, and as such it is likely constrained at multiple levels. Some of these constraints are probably introduced during the selection and encoding of scene objects and are best described as attentional and memory limitations. Other constraints are introduced during the representation and comparison of the changing objects. The present study, having proposed a computationally explicit model free of memory and attentional limitations, makes it possible to isolate the contribution of these sources of constraint in a realworld change detection task. Once the process comparing the pre- and postchange scenes is better understood, operations implementing memory and attentional constraints can then be integrated into the existing representational framework to create a more complete picture of change detection behavior. Care should be taken, however, to add these constraints only when the data require one to do so. As the present study has demonstrated, similarity-based constraints acting on the comparison operation may account for far more change 
detection behavior than has previously been thought to be the case, meaning that the role of memory and attentional constraints may have been overemphasized in the development of change detection theory.

Future work will more fully explore this relationship between the representation of change detection stimuli and the processes used to compare these representations, particularly with regard to fully realistic scenes and the role of eye movements in a change detection task (Henderson \& Hollingworth, 1999; Zelinsky, 2001a). Because even a parallel and unlimited-capacity model of change detection would be subject to visual acuity limitations, the quality of the BOLAR representation, and hence the change signal, will depend on the observer's oculomotor interaction with the change scenes. More specifically, the magnitude of the change signal will vary with the observer's current eye position, and the observer's eye position will in turn depend on the location of change signals in the BOLAR-derived difference map. Following Zelinsky (2001b, 2002), I plan to incorporate a simplified artificial retina into the BOLAR model and then use the difference signals to direct simulated gaze over the change displays. By comparing these simulated gaze movements with the oculomotor behavior of human observers viewing the identical stimuli, I hope to glean new information about the nature of the signal underlying change detection behavior.

\section{REFERENCES}

Adini, Y., Moses, Y., \& Ullman, S. (1997). Face recognition: The problem of compensating for illumination changes. IEEE Transactions on Pattern Analysis \& Machine Intelligence, 19, 721-732.

Allport, D. A. (1980). Attention and performance. In G. Claxton (Ed.), Cognitive psychology (pp. 112-153). London: Routledge \& Kegan Paul.

Amit, Y., \& Geman, D. (1997). Shape quantization and recognition with randomized trees. Neural Computation, 9, 1545-1588.

Ashby, F. G., \& LeE, W. W. (1991). Predicting similarity and categorization from identification. Journal of Experimental Psychology: General, 120, 150-172.

Ashby, F. G., \& Perrin, N. A. (1988). Toward a unified theory of similarity and recognition. Psychological Review, 95, 124-150.

Ballard, D. H., \& BRown, C. M. (1982). Computervision. Englewood Cliffs, NJ: Prentice-Hall.

Ballard, D. H., Hay hoe, M. M., Pook, P. K., \& Rao, R. P. N. (1997). Deictic codes for the embodiment of cognition. Behavioral \& Brain Sciences, 20, 723-767.

Bell, A. J., \& SeJnowski, T. J. (1997). The "independent components" of natural scenes are edge filters. Vision Research, 37, 3327-3338.

Blackmore, S. J., Brelstaff, G., Nelson, K., \& Troscianko, T. (1995). Is the richness of our visual world an illusion? Transsaccadic memory for complex scenes. Perception, 24, 1075-1081.

Bricolo,E., Poggio, T., \& Logothetis, N. (1997).3D object recognition: A model of view-tuned neurons. In M. Mozer, M. Jordan, \& T. Petsche (Eds.), Advances in neural information processing systems 9 ( $\mathrm{pp} .41$ 47). Cambridge, MA: MIT Press.

Bridgeman, B., Hendry, D., \& Stark, L. (1975). Failure to detect displacement of the visual world during saccadic eye movements. Vision Research, 15, 719-722.

Bridgeman, B., \& MAYER, M. (1983). Failure to integrate visual information from successive fixations. Bulletin of the Psychonomic Society, 21, 285-286.

Brockmole, J. R., WAnG, R. F., \& IrWIN, D. E. (2002). Temporal inte- gration between visual images and visual percepts. Journal of Experimental Psychology: Human Perception \& Performance, 28, 315 334 .

Currie, C. B., McConkie, G. W., Carlson-Radvansky, L. A., \& IRwIN, D. E. (2000). The role of the saccade target object in the perception of a visually stable world. Perception \& Psychophysics, 62, 673-683.

Duncan, J., \& Humphreys, G. W. (1989). Visual search and stimulus similarity. Psychological Review, 96, 433-458.

ECKSTEIN, M. P. (1998). The lower visual search efficiency for conjunctions is due to noise and not serial attentional processing. Psychological Science, 9, 111-118.

EdElman, S. (1993). Representing three-dimensional objects by sets of activities of receptive fields. Biological Cybernetics, 70, 37-45.

EDELMAN, S. (1998). Representation is representation of similarities. Behavioral \& Brain Sciences, 21, 449-498.

Feldman, J. A. (1985). Four frames suffice: A provisional model of vision and space. Behavioral \& Brain Sciences, 8, 265-313.

Freeman, W., \& Adelson, E. (1991). The design and use of steerable filters. IEEE Transactions on Pattern Analysis \& Machine Intelligence, 13, 891-906.

Friedman, A. (1979). Framing pictures: The role of knowledge in automatized encoding and memory for gist. Journal of Experimental Psychology: General, 108, 316-355.

GATI, I., \& TVERSKY, A. (1984). Weighting common and distinctive features in perceptual and conceptual judgments. Cognitive Psychology, 16, 341-370.

Goldstone, R. L. (1994a). Influences of categorization on perceptual discrimination. Journal of Experimental Psychology: General, 123, 178-200.

Goldstone, R. L. (1994b). The role of similarity in categorization: Providing a groundwork. Cognition, 52, 125-157.

GolDSTONE, R. L. (1994c). Similarity, interactive activation, and mapping. Journal of Experimental Psychology: Learning, Memory, \& Cognition, 20, 3-28.

Goodman, N. (1972). Seven strictures on similarity. In N. Goodman (Ed.), Problems and projects (pp. 437-447). New York: Bobbs-Merrill.

Graham, N. (1992). Breaking the visual stimulus into parts. Current Directions in Psychological Science, 1, 55-61.

Graham, N., Kramer, P., \& Yager, D. (1987). Signal-detection models for multidimensional stimuli: Probability distributions and combination rules. Journal of Mathematical Psychology, 31, 366-409.

GreEN, D., \& Swets, J. (1966). Signal detection theory and psychophysics. New York: Krieger.

GRIMES, J. (1996). On the failure to detect changes in scenes across saccades. In K. Akins (Ed.), Perception (pp. 89-110). New York: Oxford University Press.

Hayhoe, M. M., Bensinger, D. G., \& Ballard, D. H. (1998). Task constraints in visual working memory. Vision Research, 38, 125-137.

Henderson, J. M., \& Hollingworth, A. (1999). The role of fixation position in detecting scene changes across saccades. Psychological Science, 10, 438-443.

Hollingworth, A., \& Henderson, J. M. (2000). Semantic informativeness mediates the detection of changes in natural scenes. Visual Cognition, 7, 213-235.

Hubel, D. H., \& Wiesel, T. N. (1962). Receptive fields, binocular interaction and functional architecture in the cat's visual cortex. Journal of Physiology, 160, 106-154.

IRWIN, D. E. (1992). Memory for position and identity across eye movements. Journal of Experimental Psychology: Learning, Memory, \& Cognition, 18, 307-317.

IRwIN, D. E. (1996). Integrating information across saccadic eye movements. Current Directions in Psychological Science, 5, 94-99.

IRWIN, D. E., \& ANDREWS, R. V. (1996). Integration and accumulation of information across saccadic eye movements. In T. Inui \& J. McClelland (Eds.), Attention and performance XVI: Information integration in perception and communication (pp. 125-155). Cambridge, MA: MIT Press.

Irwin, D. E., Brown, J., \& Sun, J. (1988). Visual masking and visual integration across saccadic eye movements. Journal of Experimental Psychology: General, 117, 276-287. 
ITti, L., \& Koch, C. (2000). A saliency-based search mechanism for overt and covert shifts of visual attention. Vision Research, 40, 14891506 .

Kahneman, D., \& Treisman, A. (1984). Changing views of attention and automaticity. In R. Parasuraman \& D. R. Davies (Eds.), Varieties of attention (pp. 129-161). Orlando, FL: Academic Press.

Kahneman, D., Treisman, A., \& Gibbs, B. J. (1992). The reviewing of object files: Object-specific integration of information. Cognitive Psychology, 24, 175-219.

Koch, C., \& Ullman, S. (1985). Shifts of selective visual attention: Toward the underlying neural circuitry. Human Neurobiology, 4, 219 227.

Lades, M., Vorbruggen, J. C., Buhmann, J., Lange, J., von der Malsburg, C., Wurtz, R. P., \& Konen, W. (1993). Distortion invariant object recognition in the dynamic link architecture. IEEE Transactions on Computers, 42, 300-311.

LANDY, M. S., \& Movshon, J. A. (1991). Computationalmodels of visual processing. Cambridge, MA: MIT Press.

LeVIn, D. T., \& Simons, D. J. (1997). Failure to detect changes to attended objects in motion pictures. Psychonomic Bulletin \& Review, 4, 501-506.

LoGAN, G. D. (1997). The automaticity of academic life: Unconscious applications of an implicit theory. In R.S. Wyer (Ed.), Advances in social cognition (Vol. 10, pp. 157-179). Mahwah, NJ: Erlbaum.

Lu, Z-L., \& Dosher, B. A. (1999). Characterizing human perceptual inefficiencies with equivalent internal noise. Journal of the Optical Society of America A, 16, 764-778.

Malik, J., \& Perona, P. (1990). Preattentive texture discrimination with early vision mechanisms. Journal of the Optical Society of America A, 7, 923-932.

McConkie, G. W., \& Currie, C. B. (1996). Visual stability across saccades while viewing complex pictures. Journal of Experimental Psychology: Human Perception \& Performance, 22, 563-581.

McConkIE, G. W., \& RAYNER, K. (1976). Identifying the span of the effective stimulus in reading: Literature review and theories of reading. In H. Singer \& R. B. Ruddell (Eds.), Theoretical models and processes of reading (2nd ed., pp. 137-162). Newark, DE: International Reading Association.

McConKIE, G. W., \& ZoLA, D. (1979). Is visual information integrated across successive fixations in reading? Perception \& Psychophysics, 25, 221-224

Medin, D. L., Goldstone, R. L., \& Gentner, D. (1990). Similarity involving attributes and relations: Judgments of similarity and difference are not inverses. Psychological Science, 1, 64-69.

Medin, D. L., Goldstone, R. L., \& Gentner, D. (1993). Respects for similarity. Psychological Review, 100, 254-278.

Murphy, G. L., \& Medin, D. L. (1985). The role of theories in conceptual coherence. Psychological Review, 92, 289-316.

MurPhy, G. L., \& WisNIEWSKI, E. J. (1989). Categorizing objects in isolation and in scenes: What a superordinate is good for. Journal of Experimental Psychology: Learning, Memory, \& Cognition, 15, 572586.

Nosofsky, R. M. (1986). Attention, similarity, and the identificationcategorization relationship. Journal of Experimental Psychology: General, 115, 39-57.

NosOFSKY, R. M. (1992). Similarity scaling and cognitive process models. Annual Review of Psychology, 43, 25-53.

O'Regan, J. K. (1992). Solving the "Real" mysteries of visual perception: The world as an outside memory. Canadian Journal of Psychology, 46, 461-488.

O'Regan, J. K., Deubel, H., Clark, J. J., \& Rensink, R. A. (2000). Picture changes during blinks: Looking without seeing and seeing without looking. Visual Cognition, 7, 191-211.

O'Regan, J. K., Rensink, R. A., \& Clark, J. J. (1999). Change-blindness as a result of "mudsplashes." Nature, $\mathbf{3 9 8}, 34$.

Palmer, J., Ames, C. T., \& Lindsey, D. T. (1993). Measuring the effect of attention on simple visual search. Journal of Experimental Psychology: Human Perception \& Performance, 19, 108-130.

Palmer, S. E. (1978). Structural aspects of visual similarity. Memory \& Cognition, 6, 91-97.
Parker, J. R. (1993). Practical computer vision using C. New York: Wiley.

PARKER, R. E. (1978). Picture processing during recognition. Journal of Experimental Psychology: Human Perception \& Performance, 4, 284-293.

Pashler, H. (1988). Familiarity and visual change detection. Perception \& Psychophysics, 44, 369-378.

Pylyshyn, Z [W.] (1989). The role of location indexes in spatial perception: A sketch of the FINST spatial-index model. Cognition, 32, 65-97.

Pylyshyn, Z W., \& Storm, R. W. (1988). Tracking multiple independent targets: Evidence for a parallel tracking mechanism. Spatial Vision, 3, 179-197.

Raymond, J. E., Shapiro, K. L., \& Arnell, K. M. (1995). Similarity determines the attentional blink. Journal of Experimental Psychology: Human Perception \& Performance, 21, 653-662.

Rayner, K., \& Pollatsek, A. (1983). Is visual information integrated across saccades? Perception \& Psychophysics, 34, 39-48.

RENSINK, R. A. (2000a). The dynamic representation of scenes. Visual Cognition, 7, 17-42.

RENSINK, R. A. (2000b). Visual search for change: A probe into the nature of attentional processing. Visual Cognition, 7, 345-376.

Rensink, R. A. (2002). Change detection. Annual Review of Psychology, 53, 245-277.

Rensink, R. A., O'Regan, J. K., \& Clark, J. J. (1997). To see or not to see: The need for attention to perceive changes in scenes. Psychological Science, 8, 368-373.

Rohaly, A. M., Ahumada, A. J., Jr., \& Watson, A. B. (1997). Object detection in natural backgrounds predicted by discrimination performance and models. Vision Research, 37, 3225-3235.

SCHOLL, B. J. (2000). Attenuated change blindness for exogenously attended items in a flicker paradigm. Visual Cognition, 7, 377-396.

Scott-Brown, K. C., Baker, M. R., \& Orbach, H. S. (2000). Comparison blindness. Visual Cognition, 7, 253-267.

Scott-Brown, K. C., \& Orbach, H. S. (1999). Contrast discrimination, non-uniform patterns and change blindness. Proceedings of the Royal Society of London: Series B, 265, 2159-2166.

SHEPARD, R. N. (1980). Multidimensional scaling, tree-fitting, and clustering. Science, 210, 390-398.

Shepard, R. N. (1987). Toward a universal law of generalization for psychological science. Science, 237, 1317-1323.

SimONS, D. J. (2000). Current approaches to change blindness. Visual Cognition, 7, 1-15.

Simons, D. J., \& ChABRis, C. F. (1999). Gorillas in our midst: Sustained inattentional blindness for dynamic events. Perception, 28, 1059-1074.

Simons, D. J., Franconeri, S. L., \& Reimer, R. L. (2000). Change blindness in the absence of a visual disruption. Perception, 29, 11431154.

Simons, D. J., \& Levin, D. T. (1997). Change blindness. Trends in Cognitive Sciences, 1, 261-267.

Simons, D. J., \& LeVin, D. T. (1998). Failure to detect changes to people during a real-world interaction. Psychonomic Bulletin \& Review, 5, 644-649.

SPERLING, G. (1960). The information available in brief visual presentations. Psychological Monographs, 74, (11, Whole No. 498).

TownSEND, J. T. (1976). Serial and within-stage independent parallel model equivalence on the minimum completion time. Journal of Mathematical Psychology, 14, 219-238.

TownsEnd, J. T. (1990). Serial vs. parallel processing: Sometimes they look like Tweedledum and Tweedledee but they can (and should) be distinguished. Psychological Science, 1, 46-54.

Trick, L. M., \& Pylyshyn, Z W. (1993). What enumeration studies can show us about spatial attention: Evidence for limited capacity preattentive processing. Journal of Experimental Psychology: Human Perception \& Performance, 19, 331-351.

Tversky, A. (1977). Features of similarity. Psychological Review, 84, 327-352.

Wallis, G., \& Bülthoff, H. (2000). What's scene and not seen: Influences of movement and task upon what we see. Visual Cognition, $\mathbf{7}$, 175-190. 
Watanabe, S. (1985). Pattern recognition: Human and mechanical. New York: Wiley.

Werner, S., \& Thies, B. (2000). Is "change blindness" attenuated by domain-specific expertise? An expert-novices comparison of change detection in football images. Visual Cognition, 7, 163-173.

Williams, P., \& Simons, D. J. (2000). Detecting changes in novel, complex three-dimensional objects. Visual Cognition, 7, 297-322.

Wright, M., Green, A., \& BAKer, S. (2000). Limitations for change detection in multiple Gabor targets. Visual Cognition, 7, 237-252.

ZELINSKY, G. J. (1998). Detecting changes between scenes: A similaritybased theory using iconic representations (Tech. Rep. No. CNS-98-01). Urbana, IL: Beckman Institute, Cognitive Neuroscience Group.

ZELINSKY, G. J. (1999). Effects of visual similarity on change detection: A behavioral and computational analysis. Investigative Ophthalmology \& Visual Science, 40(4), S50.

ZeLINSKy, G. J. (2001a). Eye movements during change detection: Implications for search constraints, memory limitations, and scanning strategies. Perception \& Psychophysics, 63, 209-225.

ZelinsKy, G. J. (2001b). A theory of eye movements during real-world visual search. Abstracts of the Psychonomic Society, 6, 61.

ZeLiNSKY, G. J. (2002). A theory of gaze behavior during real-world visual search [Abstract]. Journal of Vision, 2(7), 726a.

ZELINSKY, G. J., \& LoSCHKY, L. (1998). Toward a more realistic assessment of visual short-term memory. Investigative Ophthalmology \& Visual Science, 39, S224.

Zelinsky, G. J., \& LoschKy, L. (2003). Forgetting what we have just seen: Serial order effects for objects in scenes revealed by eye movements. Manuscript submitted for publication.

Zipser, D., \& Andersen, R. A. (1988). A back-propagation programmed network that simulates response properties of a subset of posterior parietal neurons. Nature, 331, 679-684.

\section{NOTES}

1. Memory and attentional constraints might also be expected to interact (Rensink, 2000b;Zelinsky, 2001a). For example, if only 5 objects from a 10-item display can be simultaneously held in short-term memory, this 5-object limitation would reflect a memory constraint, whereas the specific objects selected as the elements of this subset would reflect an attentional constraint.

2. Rensink referred to the average number of items retained in memory across each change cycle as "hold," and derived a measure of hold as the cycle alternation time (the on-time of the pre- and postchange scene + the off-time blank interval) divided by the slope of the change detection RT set size function. He reasoned that the number of items held in memory might increase with display on-time up to a critical capacitydetermined point, after which further increases in on-time should no longer produce greater estimates of hold. Capacity was defined as the asymptote on this hold $\times$ on-time function.

3. The use of the terms within-category and across-category in the present context refer to the superordinate level of categorization (Murphy \& Wisniewski, 1989). Although it is also certainly reasonable to think of a hammer changing into a wrench as an across-category change, tools and toys are more widely separated in category space than either hammers and wrenches (a basic level distinction) or box wrenches and crescent wrenches (a subordinate level distinction). For this reason, I chose the superordinate level to illustrate an effect of categorical relatedness on change detection.

4. Because object changes were limited to substitutions in this study, an identity change necessarily accompanied changes in the orientation condition (and, of course, the category condition). There were no cases in which the same object simply flipped between left and right orientations. However, given that objects were randomly assigned to similar and different orientation conditions, it is unlikely that identity-specific differences between the changing objects were systematically contributing to any observed main effect of object orientation on detection performance.

5. A version of a memory-limited model can explain the observed data if one assumes the encoding of select object properties from several objects in a display rather than the encoding of several object properties from select display objects. For example, the detection of change between two similarly oriented objects might require, on average, the encoding of more properties into memory than would the detection of change between differently oriented objects. If a capacity limit was exceeded before this differentiating property could consistently be encoded into memory, performance might suffer in the similar orientation condition. However, because this explanation requires the encoding of orientation and category information for multiple display items, it is more similar in principle to a parallel processing model than to one assuming detailed working memory representations for a small number of distinct objects.

6. Of course the existence of a limited comparison operation does not mean that change detection might not also be limited by attention or memory, and it would be fairly easy to devise hybrid models exhibiting multiple sources of constraint. However, for the sake of theoretical argument, I assume in this study that attention- and memory-based constraints are not necessary to an explanation of change detection behavior. By restricting consideration to the comparison operation, I can better isolate and evaluate any potential contribution of this operation in a change detection task.

7. Many filter-based models can be created to represent real-world stimuli in a change detection task, each differing in its featural repertoire and level of complexity. It was not my intention in this study to search this space of models. I make no attempt to pit one type of model against another in order to find the optimal change detection representation. Given that no unlimited-capacity parallel model is currently being given serious consideration in the change detection literature, this level of specificity would be premature.

8. Although BOLARs are likely to be affected by visual noise, it is important not to confuse the BOLAR representation with an iconic representation (Sperling, 1960). If an iconic representation is analogous to the veridical pixel representation constituting an image, a BOLAR representation should be thought to consist of the basic visual features extracted from these pixels. Whereas an iconic representation is therefore extremely susceptible to many forms of visual interference (e.g., masks, motion artifacts, noise-induced decay, etc.), the slightly more abstracted BOLAR representation would likely be less affected by these factorspossibly surviving long enough to influence many visually mediated real-world behaviors, including change detection. 


\section{APPENDIX A}

All of the filters used in this study were derived from a circularly symmetric Gaussian of the following form:

$$
G(x, y)=e^{-\left(x^{2}+y^{2}\right)} .
$$

For example, the leftmost filter in Figure 4 can be described by taking the first derivative of this 0th order Gaussian with respect to $x$ and $y$, yielding the directional functions

$$
\frac{\partial G(x, y)}{\partial x}=-2 x e^{-\left(x^{2}+y^{2}\right)},
$$

and

$$
\frac{\partial G(x, y)}{\partial y}=-2 y e^{-\left(x^{2}+y^{2}\right)} .
$$

Similarly, the second order Gaussian (middle filter) can be described by the terms

and

$$
\frac{\partial^{2} G(x, y)}{\partial x^{2}}=-2 e^{-\left(x^{2}+y^{2}\right)}+4 x^{2} e^{-\left(x^{2}+y^{2}\right)}
$$

$$
\frac{\partial^{2} G(x, y)}{\partial y^{2}}=-2 e^{-\left(x^{2}+y^{2}\right)}+4 y^{2} e^{-\left(x^{2}+y^{2}\right)},
$$

and the third order Gaussian (rightmost filter) can be described by

$$
\frac{\partial^{3} G(x, y)}{\partial x^{3}}=4 x e^{-\left(x^{2}+y^{2}\right)}-8 x^{3} e^{-\left(x^{2}+y^{2}\right)}+8 x e^{-\left(x^{2}+y^{2}\right)}
$$

and

$$
\frac{\partial^{3} G(x, y)}{\partial y^{3}}=4 y e^{-\left(x^{2}+y^{2}\right)}-8 y^{3} e^{-\left(x^{2}+y^{2}\right)}+8 y e^{-\left(x^{2}+y^{2}\right)} .
$$

Combining like terms in the equations above produces a basis set of nine directional derivatives allowing full orientation "steerability" up to the third Gaussian order. As demonstrated by Freeman and Adelson (1991), defining a first-order derivative of a circularly symmetric Gaussian in terms of the basis functions

$$
G_{1}^{0}=\frac{\partial G(x, y)}{\partial x}=-2 x e^{-\left(x^{2}+y^{2}\right)}
$$

and

$$
G_{1}^{\pi / 2}=\frac{\partial G(x, y)}{\partial y}=-2 y e^{-\left(x^{2}+y^{2}\right)}
$$

allows any intermediate filter orientation to be derived simply by "steering" or substituting a different value for $\theta$ in the equation

$$
G_{1}^{\theta}=\cos (\theta) G_{1}^{\theta}+\sin (\theta) G_{1}^{\pi / 2} .
$$

These authors showed that the number of basis filters needed to span a particular Gaussian derivative space is one more than the order. In other words, two interpolation functions are required to span the first derivative orientation space, three to span the second, and four to span the third. The nine filter orientations used in this study were chosen from this basis set in order to enable orientation steerability. It is important to note, however, that I am currently not exploiting this steerability property, electing instead to use only the specific nine oriented filters constituting the basis set. Nevertheless, by building the present representational scheme around these particular orientations, additional oriented filters can be trivially added to the model should future tasks require a finer orientation representation. 


\section{APPENDIX B}

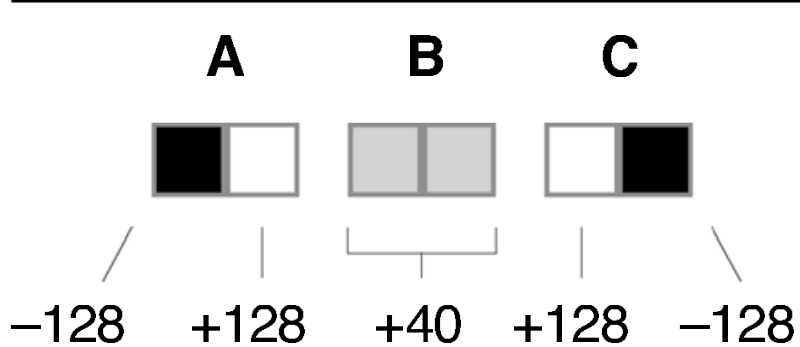

Figure B1.

The clearest way to demonstrate the concept of filtering is to consider a simplified example. The patterns illustrated in Figure B1 represent three simple objects, with each object consisting of only two pixels arranged side by side. Assume that (1) a 256-value 8-bit code is used to represent the grayscale intensity of these pixels, and (2) these 256 values are normalized within the range of -128 to +128 . The black and white pixels in patterns $\mathrm{A}$ and $\mathrm{C}$ would therefore have intensities of -128 and +128 , respectively. Also assume that the two gray pixels that make up pattern $B$ each have an intensity of +40 .

Now consider the one-dimensional filter illustrated in Figure B2. The filter spans only two pixels, giving it a "step-like" appearance. Although this two-pixel filter was customized to process the two-pixel patterns above, filters are ordinarily far smaller than the images to which they are applied. When such a size mismatch occurs, the filter must be centered over a particular pixel in the image. Often an algorithm is used to systematically move and apply the filter at each point in the image, thereby "filtering" the entire image.

The technique for applying a filter to a pattern is termed convolution. Convolution entails multiplying the value or weight of each point in the filter by the corresponding pixel value in the image. When an entire image is being filtered, these weighted pixel values are typically normalized and summed, with this sum then used to replace the pixel value in the image over which the filter is centered. The present study deals primarily with raw filter responses (the sum of the filter-weighted image pixels) and does not require this extra step of generating a fil- tered image. To compute the response of this two-pixel filter applied to pattern $\mathrm{A}$, we use

$$
\text { filter response }=\left(F_{\mathrm{L}} * P_{\mathrm{L}}\right)+\left(F_{\mathrm{R}} * P_{\mathrm{R}}\right),
$$

where $F_{\mathrm{L}}$ and $F_{\mathrm{R}}$ are the left and right filter weightings and $P_{\mathrm{L}}$ and $P_{\mathrm{R}}$ are the left and right pixel values in the pattern. Doing the arithmetic, we find a filter response of $-32,768$. Interestingly, applying the same filter to pattern $\mathrm{C}$ (rotated $180^{\circ}$ relative to pattern $\mathrm{A}$ ) results in the identical filter response (+ 32,768 ), only in the positive direction rather than the negative. This equivalent coding of response magnitude enables oriented filters spanning only $180^{\circ}$ to represent all $360^{\circ}$ of a circle. Note also that the application of this filter to pattern $B$ results in a response of 0 . The positive left component of the response is equally offset by the negative right component when summed. This insensitivity to uniform patterns enables filters to code edges and bars and other forms of spatial structure in an image.

Although the two-pixel patterns and filter considered in this example are too simple to be of use in any real-world application, the basic filtering concepts illustrated here can easily be extended to the more complex patterns and Gaussian-derivative filters used in the current model of change detection. There are several excellent texts on the topics of filtering and computer vision, and I encourage the interested reader to consult Ballard and Brown (1982) and J. R. Parker (1993) for additional details.

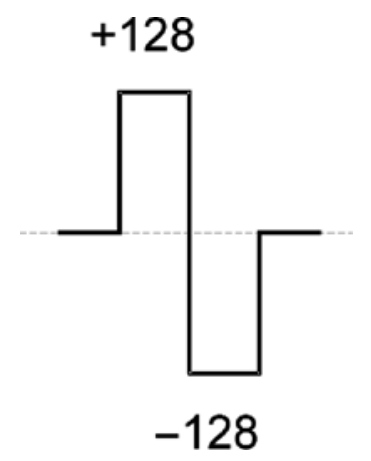

Figure B2.

(Manuscript received July 29, 1999; revision accepted for publication July 2, 2002.) 